\title{
Riqueza de especies y endemismo del componente arbóreo de la Península de Yucatán, México
}

\author{
GUILLERMO IBARRA-MANRÍQUEZ ${ }^{1}$, JOSÉ LUIS VILLASEÑOR ${ }^{1}$ Y \\ RAFAEL DURÁN GARCÍA²
}

\begin{abstract}
'Departamento de Botánica. Instituto de Biología. UNAM. Apdo. Postal 70-233. Coyoacán 04510. México, D.F. ${ }^{2}$ Centro de Investigaciones Científicas de Yucatán. Apdo. Postal 87, Cordemex, 97310, Mérida, Yucatán, México.
\end{abstract}

\begin{abstract}
Resumen. Con base en una exhaustiva consulta del Herbario Nacional de México (MEXU) y de literatura taxonómico-florística se presenta una lista actualizada de los árboles nativos de la parte mexicana de la Península de Yucatán (Campeche, Quintana Roo y Yucatán), documentando para cada especie su distribución estatal. Se reconocen 437 especies y 246 géneros, agrupados en 68 familias, con una relación adicional de especies excluidas y dudosas (308 y 68 taxa respectivamente). Las familias con números más altos de especies son Mimosaceae, Euphorbiaceae, Fabaceae, Rubiaceae y Myrtaceae, mientras que a nivel genérico los taxa más numerosos son Acacia, Eugenia, Coccoloba, Croton y Lonchocarpus. El porcentaje de endemismo es de 12.3\% (54 taxa distribuidos en 26 familias); las familias con más especies endémicas son Cactaceae, Fabaceae, Mimosaceae, Polygonaceae y Rubiaceae. A nivel estatal, Quintana Roo es la entidad que alberga una mayor riqueza (351 especies). Los resultados de cinco índices de similitud (BraunBlanquet, Dice, Drive y Kroeber, Jaccard y Simpson), tanto para el total de especies como para el elemento endémico, confirman el supuesto de que estas áreas deben de ser consideradas como parte de una misma provincia florística. Finalmente, se destaca la importancia de intensificar el trabajo florístico y taxonómico con el propósito de evaluar con mayor certeza la riqueza florística de la Península de Yucatán, y simultáneamente definir con más precisión su extensión, grado de endemismo y sus relaciones biogeográficas con regiones aledañas.

Palabras clave: árboles tropicales, diversidad de especies, endemismo, fitogeografía, Península de Yucatán.

Abstract. Based on exhaustive studies of herbarium specimens held at the National Herbarium of Mexico (MEXU), as well as of the literature, an updated list of the native species of trees of the Mexican portion of the Yucatan Peninsula is provided (Campeche, Quintana Roo y Yucatan). For each species the state(s) in which it occurs is cited. The list includes 437 species belonging to 246 genera and 68 families. An appendix listing 376 excluded species, including 68 of dubious occurrence in the,peninsula is provided, clarifying the reason for their exclusion as members of the flora of the Peninsula. The richest families are Mimosaceae, Euphorbiaceae, Fabaceae, Rubiaceae, and Myrtaceae; the richest genera are Acacia, Eugenia, Coccoloba, Croton and Lonchocarpus. The percentage of endemism is around $12.3 \%$ (54 taxa in 26 families); the families with more endemic species are Cactaceae, Fabaceae, Mimosaceae, Polygonaceae and Rubiaceae. At the state level, Quintana Roo harbors the most species (351). The use of five different similarity coefficients (Braun-Blanquet, Dice, Drive \& Kroeber, Jaccard and Simpson) to analyze both the total number of species and the endemic ones, supports the idea of considering the states as part of the same floristic province. Finally, the need to intensify the floristic and taxonomic work, aimed at evaluating in the near future more properly the floristic richness in the Peninsula is pointed out. This will allow a more precise definition of its floristic subdivisions, its degree of endemism and its floristic relationships with neighbouring regions.
\end{abstract}

Key words: tropical trees, species diversity, endemism, phytogeography, Yucatan Peninsula.

\section{INTRODUCCIÓN}

Desde hace algunas décadas, existe un claro consenso entre diversos autores en delimitar a la Península de Yucatán como unaprovincia fisiográfica, morfotectónica oflorística de México, ponderando básicamente aspectos geológicos, fisiográficos, de afinidades biogeográficas y de endemismo de la biota (Standley, 1936; Smith, 1941; Goldman y Moore, 1946; Alvarez, 1961; Barrera, 1962; Rzedowski, 1978; Leopold, 1990; Flores, 1991; Ferrusquía-Villafranca, 1993). Desde el punto de vista fitogeográfico, las primeras consideraciones sobre su flora apuntaban a resaltar que sus afinidades principales eran claramente antillanas (Lundell, 1934; Rzedowski, 1978). Actualmente esta tendencia se mantiene para el grupo de los musgos (Delgadillo, 1984), en tanto que los avances en el conocimiento de la flora vascular favorecen su parentesco con el SE de México y con Centroamérica (Miranda, 1964; Espejel, 1987; Estrada-Loera, 1991). En cuanto al grado de endemismo, Standley (1936) consideraba que el 17\% de la flora presentaba una distribución circunscrita a sus límites, aunque evaluaciones más recientes indican que $10 \%$ parece ser un porcentaje más cercano (Estrada-Loera, 1991). 
Sin duda alguna la publicación de Sosa et al. (1985) permitió resumir, ordenar y conocer con precisión el conocimiento sobre la riqueza vegetal del área. Sin embargo, después de transcurrida una década desde su publicación, la información presentada en este estudio ha disminuido su utilidad, debido principalmente al incremento en los trabajos florísticos y a los avances logrados en la sistemática de distintos grupos de plantas. De hecho, Campbell (1988) considera que el conocimiento florístico de la Península de Yucatán no es completo y la ubica como una zona prioritaria para realizar inventarios florísticos en el Neotrópico. Tomando en consideración estos puntos, el presente estudio tiene como objetivos: i) elaborar una lista actualizada de las especies arbóreas de la Península de Yucatán, ii) mencionar las especies endémicas del área, y iii) comparar la composición arbórea de los estados de Campeche, Quintana Roo y Yucatán por medio de distintos índices de similitud, para determinar si estas entidades pueden ser consideradas como parte de una misma provincia florística (sensu Rzedowski, 1978).

\section{ÁREA DE ESTUDIO}

En este trabajo se delimitó la Península de Yucatán exclusivamente a los estados mexicanos de Campeche, Quintana Roo y Yucatán $\left(17^{\circ} 50^{\prime}-21^{\circ} 35^{\prime} \mathrm{N}\right.$ y $\left.86^{\circ} 43^{\prime}-92^{\circ} 25^{\prime} \mathrm{O}\right)$, sin considerar el resto de la región biogeográfica delimitada por Barrera (1962), la cual incluye también a Belice y a la región de El Petén, en Guatemala. En México esta área se originó por una serie de levantamientos producidos durante el Mioceno y Plio-Pleistoceno y constituye una gran plataforma formada por rocas calizas de origen marino que datan del Cretácico y que disminuyen de edad desde el sur (Eoceno-Mioceno) hacia el norte (Pleistoceno-Holoceno); sus rasgos fisiográficos más peculiares son su relieve relativamente plano (altitudes menores de $400 \mathrm{msnm}$ ) y lo reducido de sus corrientes superficiales (Alvarez, 1961; Barrera, 1962; Miranda, 1964; Ferrusquía-Villafranca, 1993). Los suelos presentan una distribución muy heterogénea, aunque en términos generales pueden clasificarse en dos grupos: los xeromórficos, con drenaje deficiente y ubicados principalmente hacia la parte norte de la Península de Yucatán, y los hidromórficos, de tipo inundable o semi-inundable, presentes básicamente hacia la porción sur (Hernández-X., 1959; Orellana et al., 1985).

García (1988) indica que el clima predominante en el área es el Aw (caliente subhúmedo con lluvias de verano), aunque también se encuentra a lo largo de la franja costera de Yucatán el clima BS (seco o árido), existiendo un gradiente climático en sentido noroeste-sureste. Las temperaturas medias anuales varían entre $24^{\circ}$ y $26^{\circ}$, con oscilaciones de $5-6^{\circ}$ entre el mes más caliente (mayo) y el más frío (enero); las precipitaciones varían desde cerca de $500 \mathrm{~mm}$ en la parte norte, donde la temporada de «secas» abarca de noviembre a mayo, a cerca de $1500 \mathrm{~mm}$ en las regiones más sureñas, donde la época de menor precipitación se produce de febrero a abril (Miranda, 1964). De manera más específica, Orellana et al. (1985) reconocen seis tipos de clima para la zona: i) $\mathrm{BS}_{0}\left(\mathrm{~h}^{\prime}\right)\left(\mathrm{x}^{\prime}\right) \mathrm{i}$, seco, cálido con lluvias de verano, ii) $\mathrm{BS}_{1}\left(\mathrm{~h}^{\prime}\right)\left(\mathrm{w}^{\prime}\right) \mathrm{i}$, semiárido, cálido con lluvias de verano, iii) Aw ", ${ }_{0}\left(\mathrm{x}^{\prime}\right)(\mathrm{i}$ ')g, cálido, el más seco de los subhúmedos, con lluvias de verano y alto porcentaje de lluvia invernal, iv) Aw " ${ }_{1}\left(x^{\prime}\right)\left(i^{\prime}\right)$, cálido subhúmedo con lluvias de verano, v) Aw' ${ }_{1}$ ( $\left.\mathrm{x}^{\prime}\right)\left(\mathrm{i}^{\prime}\right) \mathrm{g}$, cálido subhúmedo con lluvias de verano y alto porcentaje de lluvia invernal, y vi) $\mathrm{Aw}_{2}{ }_{2}(\mathrm{i}) \mathrm{g}$, cálido, el más húmedo de los subhúmedos, con lluvias de verano.

Miranda (1964) describe con detalle 26 principales tipos de vegetación en la Península de Yucatán, proporcionando un mapa sobre su distribución en el área y las especies que las caracterizan. Entre las principales formaciones vegetales que menciona están: selva alta perennifolia, selva alta o mediana subperennifolia, selva alta o mediana subdecidua, selva mediana decidua, selva baja decidua, palmar, sabana, manglar, popal, tular y dunas costeras. Información más reciente sobre la vegetación puede ser encontrada en Bradburn y Darwin (1982), Cabrera et al. (1982), Rico-Gray (1982), Flores (1983), Espejel (1984, 1986), Moreno-Casasola y Espejel (1986), Olmsted y Durán (1986), Durán (1987, 1995), Moreno-Casasola (1988), Sánchez et al. (1991), Arellano-Guillermo y Serrano-Islas (1993) y Trejo-Torres et al. (1993).

El trabajo florístico de Sosa et al. (1985) enlista 1936 especies de plantas vasculares, agrupadas en 153 familias, siendo las más ricas en especies Compositae, Gramineae, Euphorbiaceae, Leguminosae y Rubiaceae. Como ya se mencionó anteriormente, el grado de endemismo es de $10 \%$ e inclusive se ha documentado la existencia de varios géneros endémicos como Asemnantha, Goldmanella, Harleya y Plagiolophus (Rzedowski, 1978; Estrada-Loera, 1991).

\section{MÉTODOS}

Este trabajo enlista las especies arbóreas localizadas en Campeche, Quintana Roo y Yucatán. Sin embargo, para catalogar a una especie como endémica se consideró más conveniente abarcar la totalidad del área biogeográfica delimitada por Barrera (1962). Por otra parte, el criterio más importante para considerar a una especie como árbol fue que los ejemplares de herbario revisados indicaran esta forma de crecimiento, independientemente del tamaño de la planta. Es necesario destacar que algunas de estas especies no se restringen a este hábito de crecimiento y pueden registrarse también como herbáceas, arbustos o incluso como arbustos con ramas trepadoras (p. ej. Lasianthaea fruticosa, Mimosa bahamensis y Rauvolfia tetraphylla). Otros criterios adicionales para decidir acerca de esta característica fueron la revisión de la literatura taxonómica y la consulta con especialistas de la flora. Mención aparte merecen las familias de monocotiledóneas Agavaceae, Arecaceae y Nolinaceae, ya que si bien sus miembros no pueden ser considerados como entidades estrictamente arbóreas, las especies incluidas semejan el hábito arborescente por su fisionomía y/o tamaño.

El listado fue elaborado con base en la revisión del material depositado en el Herbario Nacional (MEXU) y de los principales trabajos con información florística realizados hasta la fecha en el área (Millspaugh 1895, 1896, 1898; 
Millspaugh y Chase, 1904; Bequaert, 1933; Miranda, 1964; Standley, 1977; Bradburn y Darwin, 1982; Cabrera et al., 1982; Flores, 1983; Sousa y Cabrera, 1983; Lundell y Lundell, 1983; Sosa et al., 1985; Durán y Olmsted, 1987; Téllez y Cabrera, 1987; Villaseñor, 1989; Villanueva y Cabrera, 1990; Estrada-Loera, 1991; Cabrera et al., 1991; Cabrera, 1992; Quero, 1992; Arellano-Guillermo y Serrano-Islas, 1993; Durán, 1995). Cada uno de los nombres incluidos en esta lista florística fue revisado para verificar que se tratara de un nombre válido, ya sea por comunicación directa con los especialistas respectivos o por revisión de la literatura consultada, la cual incluye revisiones taxonómicas, descripciones de taxa nuevos para la ciencia o publicaciones más generales de importancia florístico-taxonómica (Cuadro 1). De forma simultánea se constató que las especies contaran con ejemplares en el acervo del herbario. Sin embargo, tres especies se incluyeron en el listado sin contar con esta última condición: Critonia hemipteropodia (Asteraceae), Harpalyce arborescens (Fabaceae) y Prockia crucis (Flacourtiaceae). Con respecto a las especies endémicas, tres especies de Celastraceae se incluyeron dentro de esta categoría, aunque es probable que trabajos sistemáticos futuros pudieran cambiar el estatus aquí definido.

La lista compila 807 especies agrupadas en 74 familias y se presenta en tres secciones arregladas cada una en orden alfabético con el propósito de facilitar su consulta: i) una

Cuadro 1. Bibliografía taxonómica consultada para la elaboración de la lista florística de los árboles de la Península de Yucatán, arreglada alfabéticamente con base en el taxón consultado.

\begin{tabular}{|c|c|c|c|}
\hline Taxon & Fuente & Taxon & Fuente \\
\hline Achatocarpaceae & Martínez-García (1985) & Machaerium & Rudd (1977) \\
\hline Agavaceae & García-Mendoza (1995) & Maclura & Zarucchi (1993) \\
\hline Annona & Lundell (1960) & Malvaceae & Fryxell (1988) \\
\hline Apocynaceae & Nowicke (1970) & Meliaceae & Pennington (1981) \\
\hline Arecaceae & Quero (1992) & Mimosa & Grether y Camargo (1993) \\
\hline Asteraceae & Villaseñor (1989) & Mimosaceae & Woodson y Schery (1950), Rico (1989, \\
\hline Astrocasia & Webster y Huft (1988), Webster (1992) & & $1991,1992,1994)$ \\
\hline Beaucarnea & Hernández (1993) & Moraceae & Burger $(1962,1977)$, Berg (1972) \\
\hline Bignoniaceae & Gentry $(1980,1982,1992)$ & Myrsine & Liogier y Martorell (1982) \\
\hline Bombax & Robyns (1963) & Myrtaceae & Sánchez-Vindas (1990) \\
\hline Boraginaceae & Nash y Moreno (1981) & Nectandra & Rohwer (1993) \\
\hline Bravaisia & Daniel (1988) & Nyctaginaceae & Fay (1980), Woodson \& Schery (1961) \\
\hline Cactaceae & Bravo-Hollis (1978) & Olacaceae & Sleumer (1984) \\
\hline Caricaceae & Badillo (1971), Moreno (1980) & Picramnia & Thomas (1988) \\
\hline Cassia & Irwin y Barneby (1982) & Pilocarpus & Kaastra (1982) \\
\hline Celastraceae & Edwin y Hou (1975) & Pinus & Perry (1991) \\
\hline Chamaedorea & Hodel (1992) & Piper & Tebbs $(1990,1993)$ \\
\hline Chrysobalanaceae & Prance $(1972,1989)$ & Plumeria & Woodson (1938) \\
\hline Cnidoscolus & Lundell (1945) & Polygonaceae & Ortiz (1994) \\
\hline Coccoloba & Howard $(1959,1960)$ & Prosopis & Burkart (1976) \\
\hline Cochlospermum & Poppendieck (1981) & Psychotria & Hamilton (1989a, 1989b, 1989c) \\
\hline Colubrina & Johnston (1971) & Rhamnaceae & Fernández (1993) \\
\hline Critonia & King y Robinson (1987) & Rinorea & Hekking (1988) \\
\hline Dendropanax & Cannon y Cannon (1989), Sosa (1979) & Rubiaceae & Steyermark (1972) \\
\hline Diphysa & Antonio y Sousa (1991) & Sabal & Zona (1990) \\
\hline Ebenaceae & Pacheco (1981) & Sapotaceae & Pennington (1990) \\
\hline Erythrina & Krukoff (1982) & Sapindaceae & Croat (1976) \\
\hline Erythroxylum & Plowman (1991) & Senna & Irwin y Barneby (1982) \\
\hline Flacourtiaceae & Sleumer $(1980)$ & Savia & Lott (1987) \\
\hline Ficus $\quad \mathrm{D}$ & De Wolf (1960), Ibarra-Manríquez y Wendt (1992) & Solanaceae & Nee $(1986,1993)$ \\
\hline Gaussia & Quero y Read (1986) & Swartzia & Cowan (1967) \\
\hline Guapira & Lundell (1962) & Thrinax & Read (1975) \\
\hline Hamelia & Elias (1976) & Triumfetta & Lay (1950) \\
\hline Harpalyce & Arroyo (1976) & Thymelaeaceae & Nevling y Barringer (1988) \\
\hline Hernandiaceae & Espejo (1991a, 1991b) & Ulmaceae & Nee (1984ab) \\
\hline Hyperbaena & Mathias y Theobald (1981) & Varios & Standley (1935), Lundell $(1945,1960)$, Standley \\
\hline Inga & Sousa (1993) & & et al. (1946-76), Bailey (1949) y León (1987) \\
\hline Jacquinia & Ståhl (1989), Stearn (1992) & Verbenaceae & Nash y Nee (1984) \\
\hline Jatropha & McVaugh (1945) & Zapoteca & Hernández (1989) \\
\hline Leucaena & Zárate (1994) & Ziziphus & Johnston (1963) \\
\hline
\end{tabular}


lista por familia y especie de los taxa reconocidos como presentes en la Península de Yucatán, donde se indica su distribución estatal, ii) una sección de taxa excluidos en la que se menciona la razón para suprimirlas de la primera sección del listado y iii) una lista de especies dudosas, resaltando el motivo por el que es incierta su presencia en el área. La distribución estatal de cada especie se obtuvo a partir de: 1) ejemplares de herbario (las abreviaturas de los estados están en negritas) y 2) por consulta bibliográfica. El arreglo de las familias para Magnoliopsida se basa en el sistema de clasificación de Cronquist (1981) y para Liliopsida en Dahlgren et al. (1985). Los nombres de la autoridades de cada especie se abreviaron de acuerdo con Brummitt y Powell (1992).

Se consideró pertinente elaborar una lista de las especies excluidas para facilitar su consulta; ésta comprende aquellos nombres de taxa que de acuerdo con el conocimiento actual y de la información de las etiquetas de herbario son: i) sinónimos, ii) nombres específicos incorrectamente citados para el área debido a determinaciones erróneas, iii) cultivares o iv) especies que, a pesar de haber sido citadas como árboles en otras regiones, dentro de la Península de Yucatán nunca han sido registradas como elementos arbóreos. Por otro lado, la lista de especies dudosas contiene básicamente aquellos taxa que carecen de material de herbario en MEXU, o bien especies pertenecientes a grupos taxonómicos muy complejos, que no cuentan con revisiones sistemáticas que permitan discernir entidades específicas bien definidas.

Finalmente, con el objeto de examinar si los estados de la Península de Yucatán pueden separarse en regiones florísticamente distintas, se evaluó la similitud florística entre las tres áreas, considerando sólo los registros de las especies obtenidos a partir de los ejemplares de herbario. Debido a la falta de consenso acerca de cuál de los numerosos índices de similitud refleja de manera más conveniente la semejanza taxonómica entre distintas localidades, se consideró pertinente retomar la propuesta de Hubálek (1982) de utilizar al menos tres distintos coeficientes de similitud, de manera que sus resultados puedan ser contrastados. Considerando los argumentos de Janson y Vegelius (1981), Hubálek (1982), Sánchez y López (1988) y Hengeveld (1990), los índices que se usaron fueron: Braun-Blanquet, Dice, Driver y Kroeber, Jaccard y Simpson. Estos algoritmos se aplicaron primeramente para el total de la flora de árboles y posteriormente para las especies endémicas. Las fórmulas de estos índices son:

$$
\begin{aligned}
& \text { Braun Blanquet }=100(\mathrm{C}) / \mathrm{N} 1 \\
& \text { Dice }=100(\mathrm{C}) / \mathrm{C}+1 / 2(\mathrm{~A}+\mathrm{B}) \\
& \text { Driver y Kroeber }=100(\mathrm{C}) /[(\mathrm{N} 1)(\mathrm{N} 2)]^{1 / 2} \\
& \text { Jaccard }=100(\mathrm{C}) / \mathrm{N} 1+\mathrm{N} 2-\mathrm{C} \\
& \text { Simpson }=100(\mathrm{C}) / \mathrm{N} 2
\end{aligned}
$$

donde $\mathrm{C}=$ número de especies en común (compartidas)

N1 = número de especies de la flora más diversa

$\mathrm{N} 2$ = número de especies de la flora menos diversa

$\mathrm{A}=$ número de especies exclusivas de la flora N1

$\mathrm{B}=$ número de especies exclusivas de la flora N2

\section{RESULTADOS}

La lista de especies de árboles de la Península de Yucatán consta de 437 especies, agrupadas en 246 géneros y 68 familias. Las familias con mayor número de elementos arbóreos son Mimosaceae, Euphorbiaceae, Fabaceae, Rubiaceae y Myrtaceae; en conjunto, las diez familias más numerosas contienen cerca del $50 \%$ de toda la flora (Cuadro 2). Este cuadro muestra también que a nivel genérico, los taxa con mayor riqueza específica resultaron ser Acacia (Mimosaceae), Eugenia (Myrtaceae), Coccoloba (Polygonaceae), Croton (Euphorbiaceae) y Lonchocarpus (Fabaceae).

Por otro lado, se encontró que existen 308 especies excluidas y 68 especies dudosas. El primer grupo puede categorizarse de la siguiente forma: 136 sinónimos, 76

Cuadro 2. Familias y géneros con mayor número de especies para los árboles de la Península de Yucatán.

\begin{tabular}{lllr}
\hline Familias & Especies & Géneros & Especies \\
\hline Mimosaceae & 39 & Acacia & 12 \\
Euphorbiaceae & 33 & Eugenia & 12 \\
Fabaceae & 28 & Coccoloba & 11 \\
Rubiaceae & 23 & Croton & 10 \\
Myrtaceae & 21 & Lonchocarpus & 9 \\
Arecaceae & 18 & Cordia & 7 \\
Polygonaceae & 15 & Ficus & 7 \\
Caesalpiniaceae & 12 & Diospyros & 6 \\
Moraceae & 12 & Pithecellobium & 6 \\
Sapotaceae & 12 & Sideroxylon & 6
\end{tabular}

determinaciones incorrectas, 50 cultivares y 46 especies pertenecientes a formas de crecimiento no arbóreas en el área (herbáceas, arbustos o lianas).

De los 437 taxa arbóreos encontrados en la Península de Yucatán, $54(12.3 \%)$ son endémicos (51 especies y 3 variedades). El Cuadro 3 muestra las 9 familias más relevantes (de un total de 26) y que engloban entre sí al 59.3\% del total de elementos endémicos. Si se compara la información de los Cuadros 2 y 3 , se puede establecer que las familias con mayor número de especies presentan un porcentaje de endemismo relativamente bajo. En cuanto a la riqueza de especies endémicas, las familias más importantes son:Cactaceae, Fabaceae, Mimosaceae, Polygonaceae y Rubiaceae. Sin embargo, es conveniente destacar que Icacinaceae y Nolinaceae son familias cuya única especie en la Península de Yucatán es endémica.

En lo referente al número de especies por estado, Quintana Roo resultó ser la entidad más diversa. Este predominio se mantiene a nivel del total de especies (351 taxa, $80.5 \%$ ), analizando exclusivamente las endémicas a la Península 
CuADro 3. Familias con mayor número de taxa endémicos y porcentaje de endemismo con respecto al total de especies en la familia para el componente arbóreo de la Península de Yucatán.

\begin{tabular}{lccc}
\hline & \multicolumn{3}{c}{ Especies } \\
\cline { 2 - 4 } Familias & Total & Endémicas & Porcentaje \\
\hline Cactaceae & 6 & 4 & 66.7 \\
Fabaceae & 28 & 4 & 14.2 \\
Mimosaceae & 39 & 4 & 10.3 \\
Polygonaceae & 15 & 4 & 26.7 \\
Rubiaceae & 23 & 4 & 17.4 \\
Arecaceae & 18 & 3 & 16.7 \\
Celastraceae & 8 & 3 & 37.5 \\
Euphorbiaceae & 33 & 3 & 9.1 \\
Sapindaceae & 10 & 3 & 30.0 \\
\hline
\end{tabular}

de Yucatán (47 taxa, 83.3\%) y considerando inclusive a las especies arbóreas con distribución restringida a una entidad (Cuadro 4). La riqueza de Campeche y Yucatán es más o menos similar, con excepción de sus especies endémicas, ya que éstas se presentan en mucha menor proporción en Campeche. El Cuadro 4 muestra que el número de especies compartidas entre las tres áreas políticas se encuentra en alrededor del $50 \%$, tanto del total de especies $(196,44.8 \%)$ como de las endémicas $(28,51.8 \%)$. De igual manera, el porcentaje de especies restringidas a un estado es también similar, tanto para el total de especies $(134,30.7 \%)$, como para los taxa endémicos $(17,31.5 \%)$.

CuAdro 4. Número de taxa arbóreos (total y endémicos) por entidad estatal en la Península de Yucatán. Entre paréntesis se indica el número de especies restringidas a cada estado.

\begin{tabular}{lcc}
\hline Estado & Total de taxa & $\begin{array}{r}\text { Endémicas a } \\
\text { la Península }\end{array}$ \\
\hline Campeche & $297(31)$ & $32(1)$ \\
Quintana Roo & $351(68)$ & $45(9)$ \\
Yucatán & $287(35)$ & $42(7)$ \\
Compartidas & 196 & 28 \\
(Cam,Qro yYuc) & &
\end{tabular}

TOTALES

436(134) y Quintana Roo son las entidades con más similitud (excepto para Braun Blanquet). Si se realiza este mismo ejercicio para los taxa endémicos, la primera tendencia continúa siendo válida, en tanto que la similitud entre las distintas entidades muestra resultados contradictorios en dependencia del algoritmo que se observe (Cuadro 6). Cabe destacar que el promedio de los cinco índices utilizados reafirma los altos coeficientes encontrados (Cuadros 5 y 6), pero no marca un panorama claro entre los estados, especialmente para los taxa endémicos, ya que las parejas Campeche-Yucatán y CampecheQuintana Roo resultaron con valores de semejanza muy cercanos.

Cuadro 5. Comparación de la riqueza arbórea entre Campeche (Cam), Quintana Roo (Qro) y Yucatán (Yuc) por medio de diferentes índices de similitud biogeográfica. En negritas se muestra el número de especies compartidas entre las parejas de estados, así como los valores más altos de los índices y del promedio.

\begin{tabular}{lllllll}
\hline & \multicolumn{6}{c}{ Índice } \\
\cline { 2 - 7 } $\begin{array}{l}\text { Pareja de } \\
\text { estados }\end{array}$ & $\begin{array}{l}\text { Braun } \\
\text { Blanquet }\end{array}$ & Dice & $\begin{array}{l}\text { Drive y } \\
\text { Kroeber }\end{array}$ & Jaccard & Simpson & Promedio \\
\hline $\begin{array}{l}\text { Cam/Qro } \\
(\mathbf{2 4 7 )}\end{array}$ & 70.4 & $\mathbf{7 6 . 2}$ & $\mathbf{7 6 . 5}$ & $\mathbf{6 1 . 6}$ & $\mathbf{8 3 . 2}$ & $\mathbf{7 3 . 6}$ \\
$\begin{array}{l}\text { Cam/Yuc } \\
(\mathbf{2 1 5})\end{array}$ & $\mathbf{7 2 . 4}$ & 73.6 & 73.6 & 58.3 & 74.9 & 70.6 \\
$\begin{array}{l}\text { Qro/Yuc } \\
(\mathbf{2 3 2})\end{array}$ & 66.1 & 72.7 & 73.1 & 57.1 & 80.8 & 70.0 \\
\hline
\end{tabular}

Cuadro 6. Comparación de la riqueza de taxa endémicos entre Campeche (Cam), Quintana Roo (Qro) y Yucatán (Yuc) usando distintos índices de similitud biogeográfica. En negritas se indica el número de taxa compartidos entre las parejas de estados, así como los valores más altos de los índices y del promedio.

\begin{tabular}{lcccccc}
\hline & \multicolumn{5}{c}{ Índice } \\
\cline { 2 - 7 } $\begin{array}{l}\text { Pareja de } \\
\text { estados }\end{array}$ & $\begin{array}{l}\text { Braun } \\
\text { Blanquet }\end{array}$ & Dice & $\begin{array}{l}\text { Drive y } \\
\text { Kroeber }\end{array}$ & Jaccard & Simpson & Promedio \\
\hline $\begin{array}{l}\text { Cam/Qro } \\
(30)\end{array}$ & 66.7 & 77.9 & 79.0 & 63.8 & $\mathbf{9 3 . 8}$ & 76.2 \\
$\begin{array}{l}\text { Cam/Yuc } \\
(\mathbf{2 9 )}\end{array}$ & 69.0 & $\mathbf{7 8 . 4}$ & $\mathbf{7 9 . 1}$ & $\mathbf{6 4 . 4}$ & 90.6 & $\mathbf{7 6 . 3}$ \\
$\begin{array}{l}\text { Qro/Yuc } \\
(\mathbf{3 4})\end{array}$ & $\mathbf{7 5 . 5}$ & 78.2 & 78.2 & 64.1 & 80.9 & 75.4 \\
\hline
\end{tabular}

\section{DISCUSIÓN}

El número de especies de árboles de la Península de Yucatán encontrado en este trabajo es menor a las 525 indicadas por Sosa et al. (1985). Esta diferencia obedece básicamente a que varios de los nombres manejados por estos autores son árboles encontrados en los tres estados de la Península de Yucatán por medio de cinco índices de similitud, muestra que existen dos tendencias claras (Cuadro 5): i) altos valores de semejanza florística entre las áreas comparadas, mayores de $65 \%$ (excepto para el Índice de Jaccard) y ii) Campeche 
actualmente considerados como sinónimos o fueron incorrectamente citados como presentes en la flora (ver la sección de especies excluidas). Adicionalmente, a pesar de que se realizó una exhaustiva revisión de literatura taxonómicoflorística y del acervo del herbario MEXU, en el listado actual queda pendiente la inclusión de varios taxa para la Península de Yucatán (ver la sección de taxa dudosos), hasta que se desarrolle a futuro un trabajo más específico en herbarios que contengan material del área (p. ej. los herbarios localizados en la Península de Yucatán) y se conozca mejor la taxonomía de algunos grupos particularmente complejos (p. ej. Nyctaginaceae).

Una situación equivalente acontece entre los árboles endémicos, ya que Estrada-Loera (1991) encontró 98 taxa, en tanto que en el listado actual este número se reduce drásticamente (54 taxa). La respuesta a este cambio de cifras tiene una marcada relación con el trabajo botánico desarrollado recientemente, el cual muestra que varios taxa ya no deben de ser considerados como endémicos, puesto que se han localizado en otras regiones fuera de la Península de Yucatán [p. ej. Bakeridesia gaumeri, Honduras (Fryxell, 1988), Cosmocalyx spectabilis, Guerrero (Gallardo, 1992), Enriquebeltrania crenatifolia, Jalisco(Lott, 1993), Parmentiera millspaughiana, Nicaragua(Gentry, 1980), Astrocasia tremula (Jamaica), Caesalpinia gaumeri (Oaxaca) y Gymnopodium floribundum (Chiapas y Oaxaca)].

Otro factor que coadyuva a esta disminución del porcentaje de endemismo es que revisiones taxonómicas recientes (p. ej. en Polygonaceae, Sapotaceae o Jacquinia) concluyen que algunas especies consideradas como endémicas son en realidad coespecíficas de taxa que tienen una distribución más amplia (ver lista de especies excluidas). Sin embargo, cabe destacar que el porcentaje obtenido se encuentra dentro del rango de endemismo que se ha calculado en trabajos precedentes (Standley, 1936; Espejel, 1987; Estrada-Loera, 1991).

Por otro lado, no parece muy sorprendente encontrar que Quintana Roo sea la entidad con mayor número de especies, ya que en la década pasada los trabajos florísticos en la entidad tuvieron un fuerte apoyo logístico, que permitieron documentar de manera más fidedigna la riqueza vegetal del estado en los acervos de varios herbarios. Esta última condición se comprueba al examinar el porcentaje de material revisado por estado (7664 ejemplares), ya que Quintana Roo abarca el 51.3\% del total, mientras que Campeche y Yucatán comparten de manera casi equitativa el porcentaje restante ( 24.1 y $24.6 \%$ respectivamente).

Considerando los valores obtenidos mediante el uso de los coeficientes de similitud usando la riqueza de especies y la flora endémica (Cuadros 5 y 6 ), se apoya la idea de que los tres estados forman parte de una misma provincia florística. Estos valores se encuentran por arriba del umbral propuesto por Sánchez y López (1988) de 66.7\% para considerar las unidades geográficas como miembros de una misma entidad fitogeográfica. En este sentido, sólo el índice de Jaccard muestra valores ligeramente menores, pero tomando en cuenta las tendencias marcadas por los otros algoritmos, tal como lo sugiere Hubálek (1982), y su promedio (Cuadros 5 y 6), los valores de Jaccard se pueden considerar también como significativos.

Adicionalmente, los resultados indican que la composición florística de los árboles de la Península de Yucatán alberga especies ampliamente distribuidas dentro de este territorio, aun en el caso del elemento endémico, ya que se comparten entre las tres entidades cerca del $50 \%$ de sus componentes (Cuadro 4). Sin embargo, conviene recordar que cerca del $30 \%$ de especies se presentan exclusivamente en una sola entidad, aunque es muy probable que futuras actividades de colecta botánica abatan estos porcentajes, ya que así lo sugiere la distribución espacial de varias especies en el área (G. Ibarra-Manríquez, en preparación).

A la luz de los resultados presentados, es importante destacar que esta contribución no proporciona un listado definitivo de la flora arbórea de la Península de Yucatán, ya que una tarea de esta envergadura requiere de la participación de más colaboradores y una labor interinstitucional más intensa. En particular, es necesaria una revisión similar a la efectuada en este trabajo para aquellos herbarios en donde se encuentre material colectado del área, especialmente los ubicados en la Península de Yucatán. Esta labor permitiría determinar la existencia de zonas deficientemente colectadas, evaluar con mayor certeza su riqueza florística y definir con más precisión su extensión, grado de endemismo y relaciones fitogeográficas con regiones aledañas. Sin duda alguna, todos estos elementos aportarían información relevante para planificar y asegurar la conservación de la riqueza florística de la flora arbórea de la Península de Yucatán.

\section{AGRADECIMIENTOS}

A los curadores del Herbario Nacional (MEXU) por las facilidades otorgadas. A los siguientes especialistas por compartir con nosotros su conocimiento sobre las plantas de la Península de Yucatán:William R. Anderson (Malpighiaceae), Salvador Arias (Cactaceae), Goreti Campos (Boraginaceae), JoséLuis Contreras (Caesalpinia), Fernando Chiang (Rutaceae), Oscar Dorado (Harpalyce), Martha Martínez(Croton), Abisaí García-Mendoza (Agavaceae), Luis Hernández(Beaucarnea), Adolfo Espejo (Hernandiaceae), Lourdes Rico (Acacia, Pithecellobium y géneros afines), Hermilo Quero (Arecaceae), Oswaldo Téllez (Leguminosae) y Sergio Zárate (Leucaena). A Patricia Dávila y Jorge Meave su incondicional apoyo logístico y académico, así como su minuciosa revisión del trabajo. Miguel Martínez-Ramos, Fernando Chiang y dos revisores anónimos aportaron importantes sugerencias que permitieron una mejor estructura y discusión del artículo. GIM agradece a la Dirección General de Asuntos del Personal Académico (DGAPA, UNAM), el goce de una beca de doctorado, así como el financiamiento otorgado por el Programa de Apoyo para Estudios de Posgrado (UNAM). 


\section{LISTADO DE LOS ÁRBOLES DE LA PENÍNSULA DE YUCATÁN}

Abreviaturas: Cam (Campeche), Qro (Quintana Roo) y Yuc (Yucatán). Simbología: * especies endémicas; ** especies endémicas que exceden ligeramente los límites definidos por Barrera (1962); † especies cultivadas; • especies no arbóreas (herbáceas, arbustos, arbustos trepadores o lianas) y sin ejemplares de herbario en MEXU.

\section{DIVISIÓN CONIFEROPHYTA}

\section{Pinaceae}

Pinus caribaea Morelet var. hondurensis (Sénécl.) Barr. \& Golf. [Qro]

\section{DIVISIÓN MAGNOLIOPHYTA CLASE MAGNOLIOPSIDA}

\section{Acanthaceae}

Bravaisia berlandieriana (Nees) T.F. Daniel [Cam, Qro,Yuc]

B. integerrima (Spreng.) Standl. [Yuc]

\section{Achatocarpaceae}

\section{Achatocarpus nigricans Triana [Cam,Qro]}

\section{Anacardiaceae}

Astronium graveolens Jacq. [Cam,Qro,Yuc]

Metopium brownei (Jacq.) Urb. [Cam,Qro,Yuc]

Mosquitoxylum jamaicense Krug \& Urb. [Qro]

Spondias mombin L. [Cam,Qro,Yuc]

\section{Annonaceae}

\section{Annona glabra L. [Cam,Qro,Yuc]}

A. reticulata L. var. primigenia (Standl. \& Steyerm.) Lundell [Cam,Qro,Yuc]

A. squamosa L. [Cam,Qro,Yuc]

Malmea depressa (Baill.) R.E. Fr. [Cam,Qro,Yuc]

Oxandra lanceolata (Sw.) Baill. [Qro]

Sapranthus campechianus (Kunth) Standl. [Cam,Qro,Yuc]

\section{Apocynaceae}

Aspidosperma megalocarpon Müll. Arg. [Cam,Qro]

Cameraria latifolia L. [Cam,Qro,Yuc]

Plumeria obtusa L. var. sericifolia (C. Wright ex Griseb.) Woodson [Cam,Qro,Yuc]

Plumeria rubra L. [Cam,Qro,Yuc]

Rauvolfia tetraphylla L. [Cam,Qro,Yuc]

Stemmadenia donnell-smithii (Rose) Woodson [Cam,Qro]

S. galeottiana (A. Rich.) Miers [Cam,Qro,Yuc]

Tabernaemontana alba Mill. [Cam,Qro]

T. amygdalifolia Jacq. [Cam,Qro,Yuc]

Thevetia ahouai (L.) DC. [Cam,Qro,Yuc]

T. gaumeri Hemsl. [Cam,Qro, Yuc]

Vallesia antillana Woodson [Qro,Yuc]

Araliaceae

Dendropanax arboreus (L.) Decne. \& Planch. [Cam,Qro,Yuc]

Asteraceae

Critonia daleoides DC. [Cam,Qro,Yuc]

* C. hemipteropodia (B.L. Rob.) R.M. King \& H. Rob. [Yuc]

* Eremosis oolepis (S.F. Blake) Gleason [Cam,Qro,Yuc]

Koanophyllon albicaulis (Sch. Bip. ex Klatt) R.M. King \& H. Rob. [Cam,Qro,Yuc]

Lasianthaea fruticosa (L.) K.M. Becker var. fruticosa [Cam,Qro,Yuc] 


\section{Bignoniaceae}

Crescentia cujete L. [Cam,Qro,Yuc]

Godmania aesculifolia (Kunth) Standl. [Cam,Yuc]

Parmentiera aculeata (Kunth) Seem. [Cam,Qro,Yuc]

P. millspaughiana L.O. Williams [Cam,Qro,Yuc]

Tabebuia chrysantha (Jacq.) Nicholson subsp. chrysantha [Cam,Qro,Yuc]

T. rosea (Bertol.) DC. [Cam,Qro,Yuc]

Tecoma stans (L.) Kunth var. stans [Cam,Qro,Yuc]

\section{Bixaceae}

Cochlospermum vitifolium (Willd.) Spreng. [Cam,Qro,Yuc]

\section{Bombacaceae}

Ceiba aesculifolia (Kunth) Britten \& E.G. Baker [Cam,Qro,Yuc]

C. pentandra (L.) Gaertn. [Cam,Qro,Yuc]

* C. schottii Britten \& E.G. Baker [Cam,Qro,Yuc]

Pachira aquatica Aubl. [Cam,Qro]

Pseudobombax ellipticum (Kunth) Dugand [Cam,Qro,Yuc]

Quararibea aff. fieldii Millsp. [Qro,Yuc]

Q. funebris (Llave) Vischer [Qro]

\section{Boraginaceae}

Bourreria oxyphylla Standl. [Qro]

* Bourreria pulchra Millsp. [Cam,Qro,Yuc]

Cordia alliodora (Ruiz \& Pav.) Oken [Cam,Qro,Yuc]

C. dentata Poir. [Cam,Yuc]

C. diversifolia Pav. ex A. DC. [Cam]

C. dodecandra A. DC. [Cam,Qro, Yuc]

C. gerascanthus L. [Cam,Qro,Yuc]

C. sebestena L. [Cam,Qro,Yuc]

C. stellifera I.M. Johnst. [Cam,Qro]

Ehretia tinifolia L. [Cam,Qro,Yuc]

Rochefortia lundellii Camp [Qro]

\section{Burseraceae}

Bursera simaruba (L.) Sarg. [Cam,Qro,Yuc]

Protium copal (Schltdl. \& Cham.) Engl. [Cam,Qro,Yuc]

\section{Buxaceae}

Buxus bartlettii Standl. [Qro]

\section{Cactaceae}

* Anisocereus gaumeri (Britton \& Rose) Backeb. [Yuc]

* Nopalea gaumeri Britton \& Rose [Cam,Qro,Yuc]

* N. inaperta Schott [Yuc]

* Pilosocereus gaumeri (Britton \& Rose) Backeb. [Yuc] Stenocereus eichlamii (Britton \& Rose) Buxb. [Yuc]

S. laevigatus (Salm-Dyck) Buxb. [Yuc]

\section{Caesalpiniaceae}

Caesalpinia gaumeri Greenm. [Cam,Qro,Yuc]

C. mollis (Kunth) Spreng. [Cam,Qro,Yuc]

C. vesicaria L. [Cam,Qro,Yuc]

* C. yucatanensis Greenm. [Cam,Qro, Yuc]

Cassia grandis L.f. [Cam,Qro, Yuc]

Cynometra retusa Britton \& Rose [Cam]

Haematoxylum campechianum L. [Cam,Qro,Yuc]

Hymenaea courbaril L. [Cam] 
Schizolobium parahybum (Vell.) S.F. Blake [Qro]

Senna atomaria (L.) Irwin \& Barneby [Cam,Qro,Yuc]

S. hayesiana (Britton \& Rose) Irwin \& Barneby [Cam,Qro]

S. racemosa (Mill.) Irwin \& Barneby var. racemosa [Cam,Qro,Yuc]

\section{Capparaceae}

Capparis cynophallophora L. [Cam,Qro,Yuc]

C. incana Kunth [Cam,Qro,Yuc]

C. indica (L.) Fawc. \& Rendle [Cam,Yuc]

C. pachaca Kunth subsp. oxysepala (C. Wright ex Radlk.) H.H. Iltis [Cam,Qro,Yuc]

C. verrucosa Jacq. [Cam,Qro]

Crataeva tapia L. [Cam,Qro,Yuc]

Forchhammeria trifoliata Radlk. [Cam,Qro,Yuc]

\section{Caricaceae}

Carica papaya L. [Cam,Qro,Yuc]

Jacaratia mexicana A. DC. [Cam,Yuc]

\section{Cecropiaceae}

Cecropia peltata L. [Cam,Qro,Yuc]

\section{Celastraceae}

Elaeodendron trichotomum (Turcz.) Lundell [Cam,Qro,Yuc]

E. xylocarpum (Vent.) DC. [Qro,Yuc]

* Maytenus belizensis Standl. [Cam,Qro,Yuc]

* M. guatemalensis Lundell [Qro]

M. phyllanthoides Benth. [Qro,Yuc]

M. schippii Lundell [Qro]

* Rhacoma gaumeri (Loes.) Standl. [Cam,Qro,Yuc]

R. puberula (Lundell) Standl. \& Steyerm. [Cam,Qro]

\section{Chrysobalanaceae}

Chrysobalanus icaco L. [Cam,Qro,Yuc]

Couepia polyandra (Kunth) Rose [Yuc]

Hirtella americana L. [Cam,Qro]

\section{Clusiaceae}

Calophyllum brasiliense Cambess. [Qro,Yuc]

Clusia flava Jacq. [Cam,Qro,Yuc]

C. rosea Jacq. [Cam]

Rheedia edulis (Seem.) Triana \& Planch. [Qro]

\section{Combretaceae}

Bucida buceras L. [Cam,Qro,Yuc]

B. spinosa (Nothrop.) Jenning. [Qro]

Conocarpus erecta L. [Cam,Qro,Yuc]

Laguncularia racemosa (L.) Gaertn. f. [Cam,Qro,Yuc]

Terminalia amazonia (J.F. Gmel.) Exell [Cam]

Dilleniaceae

Curatella americana L. [Cam]

Ebenaceae

Diospyros anisandra S.F. Blake [Cam,Qro,Yuc]

* D. bumelioides Standl. [Cam,Qro]

D. campechiana Lundell [Cam,Yuc]

D. cuneata Standl. [Cam,Qro,Yuc]

D. verae-crucis (Standl.) Standl. [Cam,Qro,Yuc] 
D. yatesiana Standl. [Cam,Qro,Yuc]

\section{Erythroxylaceae}

* Erythroxylum bequaertii Standl. [Qro,Yuc]

E. confusum Britton [Cam,Qro,Yuc]

E. havanense Jacq. [Yuc]

E. rotundifolium Lunan [Cam,Qro,Yuc]

\section{Euphorbiaceae}

Adelia barbinervis Schltdl. \& Cham. [Cam,Qro,Yuc]

A. oaxacana (Müll. Arg.) Hemsl. [Qro,Yuc]

Argythamnia guatemalensis Müll. Arg. [Qro]

Astrocasia tremula (Griseb.) Webster [Cam,Qro,Yuc]

Bernardia cf. mexicana (Hook. \& Arn.) Müll. Arg. [Cam,Yuc]

Croton arboreus Millsp. [Cam,Qro,Yuc]

* C. campechianus Standl. [Cam,Qro]

C. aff. glabellus L. [Cam,Qro,Yuc]

C. icche Lundell [Cam,Qro,Yuc]

C. lundellii Standl. [Cam,Qro,Yuc]

C. niveus Jacq. [Cam,Qro,Yuc]

C. peraeruginosus Croizat [Cam,Qro, Yuc]

C. perobtusus Lundell [Cam,Qro,Yuc]

C. reflexifolius Kunth [Cam,Qro,Yuc]

C. schiedeanus Schltdl. [Cam,Yuc]

Drypetes lateriflora (Sw.) Krug \& Urb. [Cam,Qro,Yuc]

Enriquebeltrania crenatifolia (Miranda) Rzed. [Cam,Qro,Yuc]

Euphorbia schlechtendalii Boiss. [Qro,Yuc]

Garcia nutans Vahl [Qro,Yuc]

Gymnanthes lucida Sw. [Cam,Qro,Yuc]

Hippomane mancinella $\mathrm{L}$. [Yuc]

Hura polyandra Baill. [Yuc]

* Jatropha gaumeri Greenm. [Cam,Qro,Yuc]

Margaritaria nobilis L.f. [Cam,Qro,Yuc]

Phyllanthus acuminatus Vahl [Cam,Qro,Yuc]

P. elsiae Urb. [Qro]

$P$. grandifolius L. [Qro,Yuc]

P. mocinianus Baill. [Cam,Qro,Yuc]

Sapium nitidum (Monach.) Lundell [Cam,Qro]

Savia sessiliflora (Sw.) Willd. [Yuc]

* Sebastiania adenophora Pax \& Hoffm. [Cam,Qro,Yuc]

S. confusa Lundell [Cam,Qro]

S. tikalana Lundell [Yuc]

Fabaceae

Acosmium panamense (Benth.) Yakovlev [Cam]

Andira inermis (Sw.) Kunth [Cam]

Apoplanesia paniculata C. Presl [Cam,Qro,Yuc]

Ateleia gummifera (DC.) D. Dietr. [Cam,Qro]

Dalbergia brownei (Jacq.) Urb. [Cam,Qro]

D. glabra (Mill.) Standl. [Cam,Qro,Yuc]

Diphysa carthagenensis Jacq. [Cam,Qro,Yuc]

* D. paucifoliata R. Antonio \& M. Sousa [Cam,Qro]

Erythrina caribaea Krukoff \& Barneby [Cam]

E. standleyana Krukoff [Cam,Qro,Yuc]

Gliricidia sepium (Jacq.) Kunth ex Steud. [Cam,Qro,Yuc]

Harpalyce arborescens A. Gray [Yuc]

Lonchocarpus castilloi Standl. [Cam,Qro] 
L. guatemalensis Benth. [Cam,Qro]

L. hondurensis Benth. [Cam,Qro,Yuc]

L. longistylus Pittier [Cam,Qro,Yuc]

L. luteomaculatus Pittier [Cam,Qro]

L. punctatus Kunth [Cam,Qro,Yuc]

L. rugosus Benth. [Cam,Qro,Yuc]

** L. xuul Lundell [Cam,Qro,Yuc]

* L. yucatanensis Pittier [Cam,Qro,Yuc]

Machaerium biovulatum Micheli [Cam]

Ormosia schippii Pierce ex Standl. \& Steyerm. emend. Rudd [Cam]

Piscidia piscipula (L.) Sarg. [Cam,Qro,Yuc]

* Platymiscium yucatanum Standl. [Cam,Qro,Yuc]

Pterocarpus rohrii Vahl [Qro]

Swartzia cubensis (Britton \& Wilson) Standl. var. cubensis [Cam,Qro,Yuc]

Vatairea lundellii (Standl.) Killip. ex Record [Cam]

\section{Flacourtiaceae}

Casearia aculeata Jacq. [Cam,Qro,Yuc]

C. corymbosa Kunth [Cam,Qro,Yuc]

C. emarginata C. Wright ex Griseb. [Qro,Yuc]

C. sylvestris $\mathrm{Sw}$. var. sylvestris [Qro,Yuc]

Laetia thamnia L. [Cam,Qro,Yuc]

Muntingia calabura L. [Cam,Qro,Yuc]

Prockia crucis P. Browne ex L. [Cam,Qro,Yuc]

* Samyda yucatanensis Standl. [Cam,Qro,Yuc]

Xylosma flexuosum (Kunth) Hemsl. [Cam,Qro,Yuc]

Zuelania guidonia (Sw.) Britton \& Millsp. [Cam,Qro,Yuc]

\section{Hernandiaceae}

Gyrocarpus jatrophifolius Domin [Cam,Qro,Yuc]

Hippocrateaceae

Hippocratea excelsa Kunth [Cam,Qro,Yuc]

\section{Icacinaceae}

** Ottoschulzia pallida Lundell [Qro]

\section{Lauraceae}

Licaria campechiana (Standl.) Kosterman [Cam,Yuc]

L. peckii (I.M. Johnst.) Kosterman [Cam,Qro]

Nectandra coriacea (Sw.) Griseb. [Cam,Qro,Yuc]

$N$. salicifolia (Kunth) Nees [Cam,Qro,Yuc]

Persea schiedeana Nees [Qro]

\section{Malpighiaceae}

Bunchosia lindeniana A. Juss. [Qro]

B. swartziana Griseb. [Cam,Qro,Yuc]

* Byrsonima bucidifolia Standl. [Cam,Qro,Yuc]

B. crassifolia (L.) Kunth [Cam,Qro,Yuc]

Malpighia emarginata Sessé \& Moc. ex DC. [Cam,Qro,Yuc]

M. glabra L. [Cam,Qro,Yuc]

M. lundellii Morton [Cam,Qro,Yuc]

M. souzae Miranda [Cam,Yuc]

Malvaceae

Bakeridesia gaumeri (Standl.) Bates [Qro,Yuc]

* B. yucatana (Standl.) Bates [Qro,Yuc]

* Hampea trilobata Standl. [Cam,Qro,Yuc] 
Hibiscus pernambucensis Arruda [Cam,Qro]

Thespesia populnea (L.) Solander ex Correa [Qro,Yuc]

\section{Meliaceae}

Cedrela odorata L. [Cam,Qro,Yuc]

Swietenia macrophylla King [Cam,Qro,Yuc]

Trichilia glabra L. [Qro,Yuc]

T. hirta L. [Cam,Qro,Yuc]

** T. minutiflora Standl. [Cam,Qro,Yuc]

T. moschata Sw. subsp. moschata [Cam]

T. trifolia L. subsp. trifolia [Cam]

\section{Menispermaceae}

Hyperbaena mexicana Miers [Cam,Qro,Yuc]

H. winzerlingii Standl. [Cam,Qro,Yuc]

\section{Mimosaceae}

* Acacia cedilloi L. Rico [Qro]

A. collinsii Saff. [Cam,Qro,Yuc]

A. cornigera (L.) Willd. [Cam,Qro,Yuc]

* A. dolichostachya S.F. Blake [Cam,Qro,Yuc]

* A. gaumeri S.F. Blake [Cam,Qro,Yuc]

A. gentlei Standl. [Cam,Qro]

A. globulifera Saff. [Cam,Qro,Yuc]

A. glomerosa Benth. [Cam,Qro,Yuc]

A. macracantha Humb. \& Bonpl. ex Willd. [Cam,Qro,Yuc]

A. pennatula (Schltdl. \& Cham.) Benth. [Cam,Qro,Yuc]

A. pringlei Rose [Qro,Yuc]

A. usumacintensis Lundell [Cam,Qro,Yuc]

Albizia guachapele (Kunth) Dugand [Cam]

A. niopoides (Spruce ex Benth.) Burkart [Cam,Qro]

A. purpusii Britton \& Rose [Cam]

A. tomentosa (Micheli) Standl. [Cam,Qro,Yuc]

Chloroleucon ebano (Berl.) L. Rico [Yuc]

C. mangense (Jacq.) Britton \& Rose [Cam,Qro,Yuc]

Enterolobium cyclocarpum (Jacq.) Griseb. [Cam,Qro,Yuc]

** Havardia albicans (Kunth) Britton \& Rose [Cam,Qro,Yuc]

H. pallens (Benth.) Britton \& Rose [Yuc]

H. platyloba (Spreng.) Britton \& Rose [Cam,Qro,Yuc]

Inga vera Willd. [Cam,Qro]

Leucaena leucocephala (Lam.) de Wit subsp. glabrata (Rose) Zárate [Cam,Qro]

L. leucocephala (Lam.) de Wit subsp. leucocephala [Cam,Qro,Yuc]

L. shannonii Donn. Sm. subsp. shannonii [Cam]

Lysiloma acapulcensis (Kunth) Benth. [Cam,Yuc]

L. latisiliquum (L.) Benth. [Cam,Qro,Yuc]

Mimosa bahamensis Benth. [Cam,Qro,Yuc]

Pithecellobium brownii Standl. [Cam,Qro]

P. dulce (Roxb.) Benth. [Cam,Qro,Yuc]

$P$. keyense Britton ex Coker [Cam,Qro,Yuc]

P. lanceolatum (Humb. \& Bonpl. ex Willd.) Benth. [Cam,Qro,Yuc]

$P$. pachypus Pittier [Cam,Qro]

P. unguis-cati (L.) Mart. [Cam,Qro,Yuc]

Prosopis glandulosa Torr. var. torreyana (L. Benson) I.M. Johnst. [Yuc]

P. juliflora (Sw.) DC. [Yuc]

Zygia recordii Britton \& Rose [Qro]

Zygia stevensonii (Stand1.) Record [Qro]

Moraceae

Brosimum alicastrum Sw. subsp. alicastrum [Cam,Qro,Yuc] 
Castilla elastica Cerv. subsp. elastica [Cam,Qro,Yuc]

Ficus citrifolia Mill. [Qro]

F. cotinifolia Kunth [Cam,Qro,Yuc]

F. maxima Mill. [Cam,Qro,Yuc]

F. obtusifolia Kunth [Cam,Qro]

F. ovalis (Liebm.) Miq. [Cam,Qro,Yuc]

F. pertusa L.f. [Cam,Qro,Yuc]

F. trigonata L.f. [Cam,Qro, Yuc]

Maclura tinctoria (L.) Steud. subsp. tinctoria [Cam,Qro,Yuc]

Pseudolmedia oxyphyllaria Donn. Sm. [Cam,Qro]

Trophis racemosa (L.) Urb. subsp. ramon (Schltdl. \& Cham.) W. Burger [Cam,Qro,Yuc]

\section{Myricaceae}

Myrica cerifera L. [Qro,Yuc]

\section{Myrsinaceae}

Ardisia escallonioides Schltdl. \& Cham. [Cam,Qro,Yuc]

Myrsine guianensis (Aubl.) Kuntze [Qro]

Parathesis cubana (DC.) Molinet \& M. Gómez [Cam,Qro,Yuc]

\section{Myrtaceae}

Calyptranthes lindeniana O. Berg [Qro]

C. millspaughii Urb. [Qro,Yuc]

C. pallens Griseb. var. pallens [Qro,Yuc]

Eugenia acapulcensis Steud. [Cam,Qro,Yuc]

E. aeruginea DC. [Cam,Qro]

E. axillaris (Sw.) Willd. [Cam,Qro,Yuc]

E. biflora (L.) DC. [Qro]

E. buxifolia (Sw.) Willd. [Cam,Qro,Yuc]

E. capuli (Cham. \& Schltdl.) O. Berg [Cam,Qro,Yuc]

E. aff. laevis O. Berg [Cam,Qro,Yuc]

E. laevis O. Berg var. gaumeri (Standl.) McVaugh [Qro,Yuc]

E. oerstedeana O. Berg [Qro]

** E. trikii Lundell [Cam]

E. winzerlingii Standl. [Cam,Qro,Yuc]

* E. yucatanensis Standl. [Qro,Yuc]

Myrcianthes fragans (Sw.) McVaugh var. fragans [Cam,Qro,Yuc]

Myrciaria floribunda (Willd.) O. Berg [Cam,Qro]

Pimenta dioica (L.) Merr. [Cam,Qro,Yuc]

Psidium guajava L. [Cam,Qro,Yuc]

$P$. guianeense $\mathrm{Sw}$. [Yuc]

P. sartorianum (O. Berg) Nied. [Cam,Qro,Yuc]

Ochnaceae

Ouratea lucens (Kunth) Engl. var. lucens [Cam,Qro]

O. nitida (Sw.) Engl. [Cam,Qro]

\section{Olacaceae}

Schoepfia schreberi J.F. Gmel. [Cam,Qro,Yuc]

Ximenia americana L. var. americana [Cam,Qro,Yuc]

Oleaceae

Forestiera rhamnifolia Griseb. [Qro]

\section{Polygonaceae}

Coccoloba acapulcensis Standl. [Cam,Qro,Yuc]

C. barbadensis Jacq. [Cam,Qro,Yuc]

C. belizensis Standl. [Qro]

* C. cozumelensis Hemsl. [Cam,Qro,Yuc] 
C. diversifolia Jacq. [Qro]

C. humboldtii Meisn. [Cam,Qro]

* C. reflexiflora Standl. [Cam,Qro,Yuc]

C. spicata Lundell [Cam,Qro,Yuc]

C. swartzii Meisn. [Qro]

C. uvifera L. [Cam,Qro,Yuc]

* Coccoloba sp. [Qro]

Gymnopodium floribundum Rolfe [Cam,Qro,Yuc]

* Neomillspaughia emarginata (H. Gross.) S.F. Blake [Cam,Qro,Yuc]

Podopterus mexicanus Humb. \& Bonpl. [Cam,Qro,Yuc]

Ruprechtia pallida Standl. [Cam,Qro]

\section{Rhamnaceae}

Colubrina arborescens (Mill.) Sarg. [Cam,Qro,Yuc]

C. elliptica (Sw.) Brizicky \& Stern [Qro,Yuc]

* C. greggii S. Watson var. yucatanensis M.C. Johnst. [Cam,Qro,Yuc]

C. heteroneura (Griseb.) Standl. [Yuc]

Karwinskia humboldtiana (Roem. \& Schult.) Zucc. [Cam,Qro,Yuc]

Krugiodendron ferreum (Vahl) Urb. [Cam,Qro,Yuc]

* Ziziphus yucatanensis Standl. [Qro,Yuc]

\section{Rhizophoraceae}

Rhizophora mangle L. [Cam,Qro,Yuc]

\section{Rubiaceae}

Alibertia edulis (L. Rich.) A. Rich. ex DC. [Cam,Qro]

Alseis yucatanensis Standl. [Cam,Qro, Yuc]

Antirhea lucida (Sw.) Benth. \& Hook. [Qro,Yuc]

Asemnantha pubescens Hook. f. [Cam,Qro,Yuc]

Blepharidium mexicanum Standl. [Cam]

Calycophyllum candidissimum (Vahl) DC. [Cam]

Cosmocalyx spectabilis Standl. [Qro,Yuc]

Coutarea hexandra (Jacq.) Schum. [Cam,Qro]

Exostema caribaeum (Jacq.) Roem. \& Schult. [Cam,Qro,Yuc]

E. mexicanum A. Gray [Cam,Qro,Yuc]

Guettarda combsii Urb. [Cam,Qro,Yuc]

G. elliptica Sw. [Cam,Qro,Yuc]

** G. gaumeri Standl. [Cam,Qro,Yuc]

G. macrosperma Donn. Sm. [Qro]

Machaonia acuminata Humb. \& Bonpl. [Cam,Qro]

* M. lindeniana Baill. [Cam,Qro,Yuc]

Psychotria costivenia Griseb. var. costivenia [Cam,Qro]

Randia aculeata L. [Cam,Qro,Yuc]

R. albonervia Brandegee [Cam,Yuc]

* R. longiloba Hemsl. [Cam,Qro,Yuc]

R. obcordata S. Watson [Cam,Qro,Yuc]

* R. truncata Greenm. \& Thomps. [Cam,Yuc]

Simira salvadorensis (Standl.) Steyerm. [Cam,Yuc]

\section{Rutaceae}

Ar. yris attenuata Standl. [Qro]

A. elemifera L. [Cam,Qro,Yuc]

A. sylvatica Jacq. [Qro]

Casimiroa sapota Oerst. [Qro]

C. tetrameria Millsp. [Cam,Qro,Yuc]

Esenbeckia berlandieri Baill. subsp. berlandieri [Cam,Qro,Yuc]

Pilocarpus racemosus Vahl var. racemosus [Qro]

* P. racemosus Vahl var. yucatanus Kaastra [Yuc] 
Zanthoxylum caribaeum Lam. [Cam,Qro,Yuc]

Z. fagara (L.) Sarg. [Qro,Yuc]

Z. procerum Donn. Sm. [Qro]

Salicaceae

Salix chilensis Molina [Cam]

Sapindaceae

Allophylus cominia (L.) Sw. [Cam,Qro,Yuc]

Blomia cupanioides Miranda [Qro,Yuc]

Cupania belizensis Standl. [Cam,Qro,Yuc]

C. glabra Sw. [Qro,Yuc]

* Exothea diphylla (Standl.) Lundell [Cam,Qro,Yuc]

Matayba oppositifolia (A. Rich.) Britton [Cam,Qro,Yuc]

Sapindus saponaria L. [Cam,Qro,Yuc]

* Talisia floresii Standl. [Cam,Qro,Yuc]

T. oliviformis (Kunth) Radlk. [Cam,Qro,Yuc]

* Thouinia paucidentata Radlk. [Cam,Qro,Yuc]

\section{Sapotaceae}

Chrysophyllum mexicanum Brandegee [Cam,Qro,Yuc]

Manilkara zapota (L.) van Royen [Cam,Qro,Yuc]

Pouteria campechiana (Kunth) Baehni [Cam,Qro,Yuc]

$P$. durlandii (Standl.) Baehni subsp. durlandii [Cam]

P. glomerata (Miq.) Radlk. subsp. glomerata [Yuc]

$P$. reticulata (Engl.) Eyma subsp. reticulata [Cam,Qro,Yuc]

Sideroxylon americanum (Mill.) T.D. Penn. [Cam,Qro,Yuc]

S. celastrinum (Kunth) T.D. Penn. [Cam,Qro,Yuc]

* S. foetidissimum Jacq. subsp. gaumeri (Pittier) T.D. Penn. [Cam,Qro,Yuc]

Sideroxylon obtusifolium (Roem. \& Schult.) T.D. Penn. subsp. buxifolium (Roem. \& Schult.)

T.D. Penn. [Cam,Qro,Yuc]

S. persimile (Hemsl.) T.D. Penn. subsp. persimile [Cam]

S. salicifolium (L.) Lam. [Cam,Qro,Yuc]

\section{Simaroubaceae}

Alvaradoa amorphoides Liebm. [Cam,Qro,Yuc]

Picramnia antidesma Sw. subsp. antidesma [Qro]

$P$. antidesma $\mathrm{Sw}$. subsp. fessonia (DC.) W. Thomas [Qro]

Simarouba glauca DC. [Cam,Qro,Yuc]

\section{Solanaceae}

Solanum erianthum D. Don [Cam,Qro,Yuc]

S. nudum Dunal [Cam,Qro]

\section{Sterculiaceae}

Guazuma ulmifolia Lam. [Cam,Qro,Yuc]

Theaceae

Ternstroemia tepezapote Schltdl. \& Cham. [Qro]

\section{Theophrastaceae}

* Jacquinia albiflora Lundell [Cam,Qro,Yuc]

J. arborea Vahl [Qro]

* J. flammea Millsp. ex Mez [Cam,Qro, Yuc]

J. longifolia Standl. [Qro]

J. macrocarpa Cav. subsp. macrocarpa [Cam,Qro,Yuc]

Thymelaeaceae

Daphnopsis americana (Mill.) J.R. Johnst. [Qro,Yuc] 


\section{Tiliaceae}

Heliocarpus donnell-smithii Rose [Cam,Qro,Yuc]

H. mexicanus (Turcz.) Sprague [Cam,Qro,Yuc]

Luehea candida (DC.) Mart. [Qro,Yuc]

L. speciosa Willd. [Cam,Qro,Yuc]

Trichospermum mexicanum (DC.) Baill. [Cam,Qro,Yuc]

\section{Ulmaceae}

Celtis trinervia Lam. [Cam,Yuc]

Phyllostylon brasiliense Capan. ex Benth. \& Hook. [Cam,Yuc]

Trema micrantha (L.) Blume [Cam,Qro,Yuc]

\section{Urticaceae}

Urera baccifera (L.) Gaud. [Yuc]

\section{Verbenaceae}

Avicennia germinans (L.) L. [Cam,Qro,Yuc]

Callicarpa acuminata Kunth [Cam,Qro,Yuc]

Citharexylum hirtellum Standl. [Qro]

C. schottii Greenm. [Qro,Yuc]

Cornutia grandifolia (Schltdl. \& Cham.) Schauer [Cam,Qro,Yuc]

C. pyramidata L. [Cam,Qro,Yuc]

Duranta repens L. [Cam,Qro,Yuc]

Lippia myriocephala Schltdl. \& Cham. [Cam,Yuc]

Rehdera trinervis (S.F. Blake) Mold. [Qro,Yuc]

Vitex gaumeri Greenm. [Cam,Qro,Yuc]

\section{Violaceae}

Rinorea hummelii Sprague [Cam,Qro]

\section{Zygophyllaceae}

Guaiacum sanctum L. [Cam,Qro,Yuc]

\section{CLASE LILIOPSIDA}

\section{Agavaceae}

Dracaena americana Donn. Sm. [Qro]

\section{Arecaceae}

Acoelorrhaphe wrightii (Griseb. \& H. Wendl.) H. Wendl. ex Beccari [Cam,Qro,Yuc] Acrocomia mexicana Karw. ex Mart. [Cam,Qro,Yuc]

Chamaedorea neurochlamys Burret [Cam,Qro]

C. oblongata Mart. [Cam,Qro]

* Coccothrinax readii H.J. Quero [Qro,Yuc]

Crysophila argentea Bartlett [Cam,Qro]

* Gaussia maya (O.F. Cook) H.J. Quero \& R. W. Read [Qro]

Orbignya cohune (Mart.) Dahlgren ex Standl. [Qro]

Pseudophoenix sargentii $\mathrm{H}$. Wendl. ex Sarg. [Qro,Yuc]

Roystonea dunlapiana Allen [Qro]

$R$. regia (Kunth) O.F. Cook [Yuc]

* Sabal gretheriae H.J. Quero [Qro]

S. guatemalensis Becc. [Yuc]

S. mauritiiformis (H. Karst.) Griseb. \& H. Wendl. [Qro]

S. mexicana Mart. [Cam,Qro,Yuc]

S. yapa C. Wright ex Becc. [Cam,Qro,Yuc]

Scheelea liebmannii Becc. [Cam,Qro]

Thrinax radiata Lodd. ex Schult. \& Schult. f. [Qro,Yuc] 


\section{Nolinaceae}

* Beaucarnea pliabilis (Baker) Rose [Cam,Qro,Yuc]

Addendum

Cuando este trabajo se encontraba en su edición final se consultaron algunas publicaciones que añaden dos especies arbóreas al listado del estado de Campeche. Estas especies, Ampelocera hottleii (Stand1.) Standl., Ulmaceae (Todzia, 1989) y Mosiera contrerasii (Lundell) Landrum, Myrtaceae (Landrum, 1992), no están incluidas en los análisis y resultados de este artículo.

\section{ESPECIES EXCLUIDAS}

- Acacia angustissima (Mill.) Kuntze [Mimosaceae]. Arbusto. [Cam,Qro,Yuc]

- A. farnesiana (L.) Willd. Arbusto. [Cam,Qro,Yuc]

$\dagger$ A. melanoxylon R. Br. [Yuc]

- A. riparia Kunth. Arbusto. [Cam,Qro,Yuc]

* A. sesquijuga (Britton \& Rose) Standl. Sinónimo de A. pringlei Rose.

A. spadicigera Schltdl. \& Cham. Sinónimo de A. cornigera (L.) Willd.

Abutilon gaumeri Standl. [Malvaceae]. Sinónimo de Bakeridesia gaumeri (Standl.) Bates.

A. yucatanum Standl. Sinónimo de Bakeridesia yucatana (Standl.) Bates.

Achras zapota L. [Sapotaceae]. Sinónimo de Manilkara zapota (L.) van Royen.

- Aegiphila monstrosa Mold. [Verbenaceae]. Arbusto. [Cam,Qro]

Albizia caribaea (Urb.) Britton \& Rose [Mimosaceae]. Sinónimo de A. niopoides (Spruce ex Benth.) Burkart.

$\dagger$ A. lebbeck (L.) Benth. [Cam,Qro,Yuc]

A. rubiginosa Standl. Sinónimo de Havardia albicans (Kunth) Britton \& Rose.

$\dagger$ A. saman (Jacq.) F. Muell. [Qro]

Allophylus occidentalis (Sw.) Radlk. [Sapindaceae]. Determinación incorrecta de A. cominia (L.) Sw. Allophylus occidentalis se distribuye desde México (Veracruz, Oaxaca y Chiapas) hasta el norte de Sudamérica y Las Antillas.

$\dagger$ Annona muricata L. [Annonaceae]. [Cam,Qro,Yuc]

A. primigenia Standl. \& Steyerm. Sinónimo de Annona reticulata L. var. primigenia (Standl. \& Steyerm.) Lundell.

$\dagger$ A. squamosa L. [Annonaceae]. [Cam,Qro,Yuc]

$\dagger$ Anacardium occidentale L. [Anacardiaceae]. [Cam,Yuc]

$\dagger$ Artocarpus altilis (Parkinson) Fosberg [Moraceae]. [Yuc]

Aspidosperma cruentum Woodson. [Apocynaceae]. Sinónimo de A. megalocarpon Müll. Arg.

Astrocasia phyllanthoides B. L. Rob. \& Millsp. [Euphorbiaceae]. Sinónimo de A. tremula (Griseb.) Webster.

Avicennia nitida Jacq. [Verbenaceae]. Sinónimo de A. germinans (L.) L.

- Baccharis trinervis (Lam.) Pers. var. trinervis [Asteraceae]. Arbusto. [Qro]

- Bactris balanoidea (Oerst.) H. Wendl. [Arecaceae]. Arbusto. [Cam,Qro]

- B. mexicana Mart. Arbusto. [Qro]

B. trichophylla Burret. Sinónimo de B. mexicana Mart.

* Beaucarnea ameliae Lundell [Nolinaceae]. Sinónimo de B. pliabilis (Baker) Rose.

Belotia campbellii Sprague. [Tiliaceae]. Sinónimo de Trichospermum mexicanum (DC.) Baill.

B. mexicana DC. Sinónimo de Trichospermum mexicanum (DC.) Baill.

$\dagger$ Bixa orellana L. var. orellana [Bixaceae]. [Cam,Qro,Yuc]

Bombax ellipticum Kunth [Bombacaceae]. Sinónimo de Pseudobombax ellipticum (Kunth) Dugand.

Bourreria andrieuxii (A. DC.) Hemsl. [Boraginaceae]. Determinación incorrecta de B. pulchra Millsp. Bourreria andrieuxii se distribuye desde México (Guerrero, Jalisco y Morelos) a Honduras y Nicaragua.

* B. mollis Standl. Determinación incorrecta de B. pulchra Millsp. Bourreria mollis se distribuye exclusivamente en Belice. Bravaisia tubiflora Hemsl. [Acanthaceae]. Sinónimo de B. berlandieriana (Nees) T.F. Daniel.

Bumelia americana (Mill.) Stearn [Sapotaceae]. Sinónimo de Sideroxylon americanum (Mill.) T.D. Penn.

B. celastrina Kunth. Sinónimo de Sideroxylon celastrinum (Kunth) T.D. Penn.

B. laete-virens Hemsl. Sinónimo de Sideroxylon palmeri (Rose) T.D. Penn.

B. mayana Standl. Sinónimo de Sideroxylon obtusifolium (Roem. \& Schult.) T.D. Penn. subsp. buxifolium (Roem. \& Schult.) T.D. Penn.

B. obtusifolia Roem. \& Schult. Sinónimo de Sideroxylon obtusifolium (Roem. \& Schult.) T.D. Penn. subsp. buxifolium

(Roem. \& Schult.) T.D. Penn.

B. retusa Sw. Sinónimo de Sideroxylon americanum (Mill.) T.D. Penn.

B. spiniflora A. DC. Sinónimo de Sideroxylon celastrinum (Kunth) T.D. Penn. 
Bunchosia glandulosa Turcz. [Malpighiaceae]. No se encuentra en la Península de Yucatán; se distribuye exclusivamente en Las Antillas.

B. lanceolata Turcz. Sinónimo de B. lindeniana A. Juss.

Caesalpinia cacalaco Humb. \& Bonpl. [Caesalpiniaceae]. Determinación incorrecta de C. vesicaria L. Caesalpinia cacalaco se distribuye sólo en México (Guerrero, Jalisco, Michoacán, Oaxaca y Puebla).

C. platyloba S. Watson. No se encuentra en la Península de Yucatán; se distribuye en México en Chihuahua, Colima, Jalisco, Michoacán, Nayarit, Oaxaca y Sinaloa.

†•Caesalpinia pulcherrima (L.) Sw. Arbusto. [Cam,Qro,Yuc]

C. violacea (Mill.) Standl. Este nombre se ha usado para denominar tradicionalmente a C. mollis (Kunth) Spreng. Caesalpinia violacea es un nombre taxonómicamente inválido ya que está basado en ejemplares pertenecientes al género Lonchocarpus (Fabaceae).

Carica cauliflora Jacq. [Caricaceae]. No se encuentra en la Península de Yucatán; se distribuye desde México (Chiapas, Oaxaca, Puebla, Sinaloa y Veracruz) a Colombia, Venezuela y Trinidad.

C. pennata Heilborn. Sinónimo de C. cauliflora Jacq.

Casearia guianensis (Aubl.) Urb. [Flacourtiaceae]. No se encuentra en la Península de Yucatán; se distribuye en Las Antillas, Panamá y Colombia hasta Brasil.

C. nitida Jacq. No se encuentra en la Península de Yucatán; se distribuye exclusivamente en Bahamas, Cuba, Jamaica y República Dominicana.

C. subsessiliflora Lundell. Sinónimo de C. sylvestris Sw. subsp. sylvestris.

Cassia alata L. [Caesalpiniaceae]. Sinónimo de Senna alata (L.) Roxb.

C. emarginata L. Sinónimo de Senna bicapsularis (L.) Roxb. var. bicapsularis.

$\dagger$ C. fistula L. [Qro,Yuc]

C. fruticosa Mill. Sinónimo de Senna fruticosa (Mill.) Irwin \& Barneby.

C. glauca Lam. Sinónimo de Senna sulfurea (Collad.) Irwin \& Barneby.

C. hayesiana (Britton \& Rose) Standl. Sinónimo de Senna hayesiana (Britton \& Rose) Irwin \& Barneby.

$\dagger$ C. javanica L. var. indochinensis Gagnep. [Qro]

C. pallida Vahl. Sinónimo de Senna pallida (Vahl) Irwin \& Barneby var. pallida.

C. racemosa Mill. Sinónimo de Senna racemosa (Mill.) Irwin \& Barneby.

C. villosa Mill. Sinónimo de Senna villosa (Mill.) Irwin \& Barneby.

$\uparrow$ Casuarina equisetifolia L. [Casuarinaceae]. [Cam,Qro,Yuc]

Cecropia obtusifolia Bertol. [Cecropiaceae]. Determinación incorrecta de C. peltata L. Cecropia obtusifolia se distribuye desde México (Chiapas, Colima, Guerrero, Jalisco, Oaxaca, San Luis Potosí, Sinaloa, Tabasco, Tamaulipas y Veracruz) al norte de Sudamérica.

Cedrela mexicana M. Roem. [Meliaceae]. Sinónimo de C. odorata L.

- Celtis iguanaea (Jacq.) Sarg. [Ulmaceae]. Arbusto trepador o liana. [Cam,Qro,Yuc]

* Cephalocereus gaumeri Britton \& Rose [Cactaceae]. Sinónimo de Pilosocereus gaumeri (Britton \& Rose) Backeb.

Cereus griseus Haw. Sinónimo de Stenocereus griseus (Haw.) Buxb.

C. yucatanensis Standl. Sinónimo de Anisocereus gaumeri (Britton \& Rose) Backeb.

- Cestrum diurnum L. [Solanaceae]. Arbusto. [Qro]

- C. nocturnum L. Arbusto. [Cam,Qro,Yuc]

Chamaedorea erumpens H.E. Moore [Arecaceae]. Sinónimo de C. seifrizii Burret.

- C. seifrizii Burret. Arbusto. [Cam]

- Chiococca alba (L.) Hitch. [Rubiaceae]. Herbácea o arbusto trepador. [Cam,Qro,Yuc]

Chlorophora tinctoria (L.) Gaud. ex B.D. Jacks. [Moraceae]. Sinónimo de Maclura tinctoria (L.) Steud. subsp. tinctoria

$\dagger$ Chrysophyllum cainito L. [Sapotaceae]. [Cam,Qro,Yuc]

C. oliviforme L. Especie ausente de la Península de Yucatán; se distribuye exclusivamente en Florida y Las Antillas.

- Citharexylum hexangulare Greenm. [Verbenaceae]. Arbusto. [Qro,Yuc]

$\dagger$ Citrus aurantifolia (Christm.) L.D. Swingle [Rutaceae]. [Qro]

$\dagger$ C. aurantium L. [Rutaceae]. [Yuc]

$\dagger$ C. limon (L.) Burm. [Qro,Yuc]

$\dagger$ C. limettoides Tanaka. [Yuc]

$\dagger$ C. paradisi Macfad. [Yuc]

$\dagger$ C. reticulata Blanco. [Yuc]

$\dagger$ C. sinensis (L.) Osbeck. [Yuc]

+ Clerodendrum fragrans Vent. [Verbenaceae]. [Yuc]

- C. ligustrinum (Jacq.) R. Br. Arbusto. [Qro,Yuc]

- Clidemia octona (Bonpl.) L.O. Williams [Melastomataceae]. Arbusto. [Qro] 
Clusia salvinii Donn. Sm. [Clusiaceae]. Determinación incorrecta de C. flava Jacq. Clusia salvinii se distribuye desde México (Chiapas, Michoacán, Oaxaca y Veracruz) a Belice, Guatemala y Honduras.

Coccoloba acuminata Kunth [Polygonaceae]. Determinación incorrecta de C. cozumelensis Hemsl. Coccoloba acuminata se distribuye desde Panamá a Brasil, Perú y Venezuela.

C. browniana Standl. Sinónimo de C. acapulcensis Standl.

C. floribunda (Benth.) Lindau. Sinónimo de C. venosa L.

C. hondurensis Lundell. No se encuentra en la Península de Yucatán; se distribuye en México (Oaxaca y Veracruz), Belice, Guatemala y Honduras.

C. aff. laurifolia Jacq. (fide Sousa y Cabrera, 1983). Sinónimo de C. diversifolia Jacq.

* C. lundellii Standl. Sinónimo de C. x lundellii Standl.

C. montana Standl. No se encuentra en la Península de Yucatán; se distribuye en México (Chiapas), Belice, Guatemala

y El Salvador.

C. schiediana Lindau. Sinónimo de C. barbadensis Jacq.

* C. schippii Lundell. Sinónimo de C. montana Standl.

C. venosa L. No se encuentra en la Península de Yucatán; se distribuye desde México (Chiapas, Colima, Jalisco y Oaxaca) a Nicaragua y Costa Rica.

$\dagger$ Cocos nucifera L. [Arecaceae]. [Cam,Qro,Yuc]

Colubrina reclinata (L'Hér.) Brongn. [Rhamnaceae]. Determinación incorrecta de $C$. elliptica (Sw.) Brizicky \& Stern. Fernández (1993) no menciona esta especie en su tratamiento para Rhamnaceae de México.

Conocarpus erecta L. var. sericea DC. [Combretaceae]. Esta variedad se caracteriza por tener hojas densamente sericeo pubescentes y no parece claramente distinta de $C$. erecta var. erecta, la cual tiene hojas glabras o escaso pubescentes; en consecuencia, ambas categorías subespecíficas no se reconocen en este trabajo.

Cornutia latifolia (Kunth) Mold. [Verbenaceae]. Sinónimo de C. pyramidata L.

Crataeva glauca Lundell [Capparaceae]. Sinónimo de C. tapia L. var. glauca (Lundell) Standl. \& Steyerm.

C. tapia L. var. glauca (Lundell) Standl. \& Steyerm. Esta variedad se caracteriza por tener folíolos glaucos, pero debido

a la dificultad de reconocer este aspecto en ejemplares herborizados, no se le reconoce en este trabajo.

- Critonia morifolia (Mill.) R.M. King \& H. Rob. [Asteraceae]. Arbusto. [Cam,Qro]

- Crossopetalum eucymosa (Loes. \& Pittier) Lundell. [Celastraceae]. Arbusto. [Cam,Qro]

C. parviflorum (Hemsl.) Lundell. Determinación incorrecta de C. eucymosa (Loes. \& Pittier) Lundell.

Crossopetalum parviflorum presenta problemas para su delimitación específica y no es posible proporcionar su área de distribución.

- Croton chichenensis Lundell [Euphorbiaceae]. Herbácea o arbusto. [Cam,Qro,Yuc]

C. nitens Sw. Sinónimo de C. glabellus L.

Cupania rufescens Triana \& Planch. [Sapindaceae]. Determinación incorrecta de C. belizensis Standl. Cupania rufescens se distribuye aparentemente desde México hasta Brasil y Venezuela.

C. schippii Standl. Sinónimo de C. rufescens Triana \& Planch.

$\dagger$ Cupressus lindleyi Klotzsch [Cupressaceae]. [Cam,Qro,Yuc]

Cymbopetalum sp. [Annonaceae]. Determinación incorrecta de Sapranthus campechianus (Kunth) Standl.

Daphnopsis mollis (Cham. \& Schltdl.) Standl. [Thymelaeaceae]. Determinación incorrecta de D. americana (Mill.) J.R.

Johnst. Daphnopsis mollis se distribuye sólo en México (Hidalgo, San Luis Potosí y Veracruz).

$\dagger$ Delonix regia (Bojer) Raf. [Caesalpiniaceae]. [Cam,Qro,Yuc]

$\uparrow$ Dioon spinulosum Dyer [Cycadaceae]. [Cam,Qro,Yuc]

Diospyros conzattii Standl. [Ebenaceae]. Determinación incorrecta de D. verae-crucis (Stand1.) Standl. Diospyros

conzattii presenta problemas para su delimitación específica y no es posible proporcionar su área de distribución.

D. nicaraguensis (Stand1.) Standl. Sinónimo de D. verae-crucis (Stand1.) Standl.

D. yucatanensis Lundell. Sinónimo de D. verae-crucis (Standl.) Standl.

Dipholis salicifolia (L.) A. DC. [Sapotaceae]. Sinónimo de Sideroxylon salicifolium (L.) Lam.

Diphysa macrophylla Lundell [Fabaceae]. Determinación incorrecta de D. paucifoliata R. Antonio y M. Sousa. Diphysa macrophylla se distribuye exclusivamente en Oaxaca y Veracruz.

Duranta erecta L. [Verbenaceae]. Sinónimo de D. repens L.

Erythrina berteroana Urb. [Fabaceae]. No se encuentra en la Península de Yucatán; se distribuye desde México (Chiapas

y Veracuz), hasta Colombia, Venezuela y Las Antillas.

Erytroxylum belizense Lundell [Erythroxylaceae]. Determinación incorrecta de E. bequaertii Standl. y estrechamente relacionada con E. areolatum L. E. belizense se ha indicado para Belice.

E. brevipes DC. Sinónimo de E. rotundifolium Lunan.

Esenbeckia pentaphylla (Macfad.) Griseb. [Rutaceae]. No se encuentra en la Península de Yucatán; se distribuye en Belice, Guatemala, Panamá, Colombia y Jamaica. 
E. yaaxhokob Lundell. Sinónimo de E. berlandieri Baill. ex Hemsl. subsp. berlandieri.

Eugenia flavifolia Standl. [Myrtaceae]. Sinónimo de E. farameoides A. Rich.

E. farameoides A. Rich. No se encuentra en la Península de Yucatán; se distribuye desde México (Oaxaca) hasta Belice,

Guatemala y Honduras.

E. fragans (Sw.) Willd. Sinónimo de Myrcianthes fragans (Sw.) McVaugh.

E. gaumeri Standl. Sinónimo de E. laevis O. Berg var. gaumeri (Standl.) McVaugh.

E. jambos L. Sinónimo de Syzygium jambos (L.) Alston.

E. karwinskiana O. Berg. Determinación incorrecta de E. yucatanensis Standl. Eugenia karwinskiana se distribuye en México (Hidalgo, San Luis Potosí y Tabasco) y Guatemala.

* E. mayana Standl. Sinónimo de E. buxifolia (Sw.) Willd.

Eupatorium albicaule Sch. Bip. ex Klatt [Asteraceae]. Sinónimo de Koanophyllon albicaulis (Sch. Bip. ex Klatt) R.M. King \& H. Rob.

E. daleoides (DC.) Hemsl. Sinónimo de Critonia daleoides DC.

* E. hemipteropodum B.L. Rob. Sinónimo de Critonia hemipteropodia (B.L. Rob.) R.M. King \& H. Rob.

$\dagger$ Ficus carica L. [Moraceae]. [Cam,Qro,Yuc]

$\dagger$ F. elastica Roxb. [Cam,Qro,Yuc]

F. glaucescens (Liebm.) Miq. Sinónimo de F. maxima Mill.

F. goldmanii Humb. \& Bonpl. ex Willd. No se encuentra en la Península de Yucatán; se distribuye desde México (básicamente hacia la vertiente del Pacífico desde Durango y Nayarit hasta Chiapas), Belice y Guatemala hasta Panamá.

$F$. involuta (Liebm.) Miq. Sinónimo de F. obtusifolia Kunth.

$F$. laevigata Vahl. Sinónimo de $F$. citrifolia Mill.

F. lapathifolia (Liebm.) Miq. No se encuentra en la Península de Yucatán; se distribuye en Chiapas, Oaxaca, Tabasco y Veracruz.

F. mexicana (Miq.) Miq. Sinónimo de F. maxima Mill.

$F$. radula Willd. Sinónimo de $F$. maxima Mill.

$\dagger F$. retusa $\mathrm{L}$. [Cam,Qro,Yuc]

F. tecolutensis (Liebm.) Miq. Determinación incorrecta de F. pertusa L.f. Ficus tecolutensis se distribuye desde México (Chiapas, Oaxaca y Veracruz) hasta Belice y Guatemala.

$F$. yucatanensis Standl. Sinónimo de F. trigonata L.f.

Gymnopodium antigonoides (B.L. Rob.) S.F. Blake [Polygonaceae]. Sinónimo de G. floribundum Rolfe

G. floribundum Rolfe var. antigonoides (B.L. Rob.) Standl. \& Steyerm. Sinónimo de G. floribundum Rolfe.

* G. ovatifolium (B.L. Rob.) S.F. Blake. Sinónimo de G. floribundum Rolfe.

Gyrocarpus americanus Jacq. [Hernandiaceae]. Determinación incorrecta de G. jatrophifolius Domin. Gyrocarpus americanus se distribuye exclusivamente en Centroamérica (Honduras, Nicaragua) y Sudamérica (Colombia y Venezuela).

- Hamelia patens Jacq. var. patens [Rubiaceae]. Arbusto. [Cam,Qro,Yuc]

Hampea integerrima Schltdl. [Malvaceae]. No se encuentra en la Península de Yucatán; especie endémica a Veracruz.

Harpalyce formosa Moc. \& Sessé ex DC. var. formosa [Fabaceae]. No se encuentra en la Península de Yucatán; se distribuye en México (Chiapas, Oaxaca y Puebla), Guatemala y Honduras.

H. rupicola Donn. Sm. Especie arbustiva que no se encuentra en la Península de Yucatán; se distribuye en Guatemala y Honduras.

Heliabravoa chende (Gosselin) Backeb. [Cactaceae]. No se encuentra en la Península de Yucatán; se distribuye en Oaxaca y Puebla.

Hemiangium excelsum (Kunth) A.C. Sm. [Hippocrateaceae]. Sinónimo de Hippocratea excelsa Kunth.

- Heteropterys laurifolia (L.) A. Juss. [Malpighiaceae]. Liana. [Qro]

- Hintonia octomera Bullock [Rubiaceae]. Arbusto o arbusto trepador. [Cam,Qro,Yuc]

- Indigofera suffruticosa Mill. [Fabaceae]. Arbusto. [Cam,Qro,Yuc]

$\dagger$ Inga paterno Harms [Mimosaceae]. [Qro,Yuc]

I. vera Willd. subsp. spuria (Willd.) J. León. Sinónimo de I. vera Willd.

Jacquinia aurantiaca Aiton [Theophrastaceae]. Sinónimo de J. macrocarpa Cav. subsp. macrocarpa

J. axillaris Oerst. Sinónimo de J. macrocarpa Cav. subsp. macrocarpa

J. macrocarpa Cav. subsp. pungens (A. Gray) Ståhl. No se encuentra en la Península de Yucatán; se distribuye en Guerrero, Jalisco y Michoacán.

J. paludicola Standl. No se encuentra en la Península de Yucatán; se distribuye en Belice y Guatemala.

J. pungens A. Gray. Sinónimo de J. macrocarpa Cav. subsp. pungens (A. Gray) Ståhl. 


\section{* J. schippii Standl. Sinónimo de J. longifolia Standl.}

Karwinskia calderoni Standl.[Rhamnaceae]. Determinación incorrecta de K. humboldtiana (Roem. \& Schult.) Zucc. Karwinskia calderoni se distribuye de México (Chiapas, Guerrero y Oaxaca) a Honduras y Nicaragua.

Lonchocarpus parviflorus Benth. [Fabaceae]. Determinación incorrecta de L. yucatanensis Pittier. Para Lonchocarpus parviflorus se encontraron colectas exclusivamente para Costa Rica y Nicaragua.

Lucuma hypoglauca Standl. [Sapotaceae]. Sinónimo de Pouteria glomerata (Miq.) Radlk. subsp. glomerata.

Lysiloma bahamensis Benth. [Mimosaceae]. Sinónimo de L. latisiliquum (L.) Benth.

Malpighia incana Mill. [Malpighiaceae]. Determinación incorrecta de M. souzae Miranda. Malpighia incana presenta problemas para su delimitación específica y no es posible proporcionar su área de distribución.

M. punicifolia L. Sinónimo de M. glabra L.

- Malvaviscus arboreus Cav. var. arboreus [Malvaceae]. Arbusto. [Cam,Qro,Yuc]

- M. arboreus Cav. var. mexicanus Schltdl. Arbusto. [Cam,Qro,Yuc]

$\dagger$ Mammea americana L. [Clusiaceae]. [Qro,Yuc]

- Manihot carthagenensis (Jacq.) Müll. Arg. [Euphorbiaceae]. Arbusto. [Cam,Yuc]

$\dagger$ Mangifera indica L. [Anacardiaceae]. [Qro,Yuc]

Manilkara achras (Mill.) Fosberg [Sapotaceae]. Sinónimo de M. zapota (L.) van Royen.

* M. breviloba Gilly. Sinónimo de M. zapota (L.) van Royen.

* M. staminodella Gilly. No se encuentra en la Península de Yucatán; se distribuye en Belice, Guatemala, Nicaragua y Costa Rica.

* M. striata Gilly. Sinónimo de M. zapota (L.) van Royen.

Mastichodendron capiri (A. DC.) Cronquist. Sinónimo de Sideroxylon capiri (DC.) Pittier subsp. tempisque (Pittier) T.D. Penn.

* M. foetidissimum Jacq. subsp. gaumeri (Pittier) T.D. Penn. Sinónimo de Sideroxylon foetidissimum Jacq. subsp. gaumeri

(Pittier) T.D. Penn.

M. gaumeri (Pittier) Lundell. Sinónimo de Sideroxylon foetidissimum Jacq. subsp. gaumeri (Pittier) T.D. Penn.

$\uparrow$ Melia azederach L. [Meliaceae]. [Cam,Yuc]

$\uparrow$ Melicoccus bijugatus Jacq. [Sapindaceae]. [Yuc]

- Miconia hyperprasina Naudin [Melastomataceae]. Arbusto. [Qro]

Mimosa hemiendyta Rose \& B.L. Rob. [Mimosaceae]. Sinónimo de M. bahamensis Benth.

$\uparrow$ Moringa oleifera Lam. [Moringaceae]. [Cam,Qro,Yuc]

$\uparrow$ Murraya paniculata (L.) Jacq. [Rutaceae]. [Qro,Yuc]

$\uparrow$ Myroxylon balsamum (L.) Harms var. pereirae (Royle) Harms [Fabaceae]. [Cam]

Nectandra sanguinea Rottb. [Lauraceae]. No se encuentra en la Península de Yucatán; se distribuye exclusivamente en

Sudamérica (Brasil, Guyana, Surinam y Venezuela).

Nolina pliabilis (Baker) Lundell [Nolinaceae]. Sinónimo de Beaucarnea pliabilis (Baker) Rose

* Opsiandra maya O.F. Cook [Arecaceae]. Sinónimo de Gaussia maya (O.F. Cook) H.J. Quero \& Read

$\dagger$ Parkinsonia aculeata L. [Caesalpiniaceae]. [Qro,Yuc]

$\dagger$ Persea americana Mill. var. americana [Lauraceae]. [Cam,Qro,Yuc]

Phoebe longicaudata Lundell. No se encuentra en la Península de Yucatán y su distribución es incierta debido a su complejo estatus taxonómico.

Phyllanthus nobilis (L.f.) Müll. Arg. [Euphorbiaceae]. Sinónimo de Margaritaria nobilis L.f.

Phyllostylon rhamnoides (Poiss.) Taub. [Ulmaceae]. Sinónimo de P. brasiliense Capan. ex Benth. \& Hook.

- Physalis arborescens L. [Solanaceae]. Arbusto. [Cam,Yuc]

Picramnia andicola Tul. [Simaroubaceae]. Sinónimo de P. antidesma Sw. subsp. fessonia (DC.) W. Thomas

P. teapensis Tul. No se encuentra en la Península de Yucatán; se distribuye desde México (Veracruz y Chiapas) hasta Panamá y Venezuela.

Pileus mexicanus (A. DC.) I.M. Johnst. [Caricaceae]. Sinónimo de Jacaratia mexicana A. DC.

- Piper amalago L. [Piperaceae]. Arbusto. [Cam,Qro]

- P. auritum Kunth. Arbusto. [Cam,Qro,Yuc]

P. gaumeri Trel. Sinónimo de P. amalago L.

$P$. lundellii Trel. Sinónimo de P. amalago L.

- P. marginatum Jacq. Arbustc. [Cam,Qro, Yuc]

P. psilorhachis C. DC. Determinación incorrecta de P. amalago L. Piper psilorhachis presenta problemas para su delimitación específica y no es posible proporcionar su área de distribución.

P. sempervirens (Trel.) Lundell. Determinación incorrecta de P. amalago L. Piper sempervirens presenta problemas para

su delimitación específica y no es posible proporcionar su área de distribución.

- P. yucatanense C. DC. Arbusto. [Cam,Qro]

- Pisonia aculeata L. [Nyctaginaceae]. Arbusto trepador o liana. [Cam,Qro,Yuc] 
* Pithecellobium albicans (Kunth) Benth. [Mimosaceae]. Sinónimo de Havardia albicans (Kunth) Britton \& Rose

$P$. calostachys Standl. Sinónimo de P. insigne Micheli.

P. cognatum (Schltdl.) Benth. Sinónimo de Zygia cognata (Schltdl.) Britton \& Rose.

$P$. disciferum Lundell. Sinónimo de Zygia recordii Britton \& Rose.

P. ebano (Berl.) Muller. Sinónimo de Chloroleucon ebano (Berl.) L. Rico.

- P. hymeneaefolium (Humb. \& Bonpl. ex Willd.) Benth. Arbusto. [Qro]

- $P$. insigne Micheli. Arbusto. [Cam]

P. latifolium (L.) Benth. Nombre incorrectamente aplicado a Zygia cognata (Schltdl.) Britton \& Rose.

P. leucospermum Brandegee. Sinónimo de Chloroleucon mangense (Jacq.) Britton \& Rose var. leucospermum (Brandegee)

Barneby \& Grimes.

P. mangense Macbr. Sinónimo de Chloroleucon mangense (Jacq.) Britton \& Rose.

P. microstachyum Standl. Determinación incorrecta de P. lanceolatum (Humb. \& Bonpl. ex Willd.) Benth. Pithecellobium microstachyum presenta problemas para su delimitación específica y no es posible proporcionar su área de distribución.

P. pallens (Benth.) Standl. Sinónimo de Havardia pallens (Benth.) Britton \& Rose.

P. platylobum (Spreng.) Urb. Sinónimo de Havardia platyloba (Spreng.) Britton \& Rose.

* P. recordii (Britton \& Rose) Standl. Sinónimo de Zygia recordii Britton \& Rose.

P. saman (Jacq.) Benth. Sinónimo de Albizia saman (Jacq.) F. Muell.

* P. stevensonii (Standl.) Standl. \& Steyerm. Sinónimo de Zygia stevensonii (Standl.) Record.

Plumeria alba L. [Apocynaceae]. No se encuentra en la Península de Yucatán; se distribuye exclusivamente en Las Antillas.

P. rubra L. Especie constituida por cuatro formas (f. acutifolia (Poiret) Woodson, f. lutea (Ruiz \& Pav.) Woodson, f. rubra

y f. tricolor (Poiret) Woodson), las cuales se diferencian por el color de sus flores. El reconocimiento de esta característica en los ejemplares de herbario no siempre es posible, por lo que se consideró impráctico su reconocimiento y únicamente se acepta el estatus específico; las variedades se han reportado de Quintana Roo y Yucatán.

Pouteria belizensis (Standl.) Cronquist [Sapotaceae]. No se encuentra en la porción mexicana de la Península de Yucatán; se distribuye en Tabasco, Belice y Guatemala.

$P$. chiricana (Kunth) Baehni. No se encuentra en la Península de Yucatán; especie endémica de Panamá.

*.P. gallifructa Cronquist. Sinónimo de P. torta (Mart.) Radlk. subsp. gallifructa (Cronquist) T.D. Penn.

* P. lundellii (Standl.) L.O. Williams. Sinónimo de P. belizensis (Standl.) Cronquist.

P. mammosa (L.) Cronquist. Sinónimo de P. sapota (Jacq.) H. Moore \& Stearn.

P. torta (Mart.) Radlk. subsp. gallifructa (Cronquist) T.D. Penn. No se encuentra en la Península de Yucatán; se distribuye en Belice, Guatemala y Costa Rica.

P. unilocularis (Donn. Sm.) Baehni. Sinónimo de P. reticulata (Engl.) Eyma subsp. reticulata.

Psidium yucatanense Lundell [Myrtaceae]. Sinónimo de P. sartorianum (O. Berg) Nied.

P. molle Bertol. Sinónimo de P. guianeense Sw.

Psychotria chagrensis Standl. [Rubiaceae]. Determinación incorrecta de P. fruticetorum Standl. Psychotria chagrensis se distribuye en México (Veracruz) a Costa Rica, Guatemala, Nicaragua y Panamá.

- P. fruticetorum Standl. Herbácea o arbusto. [Cam,Qro,Yuc]

P. graciliflora (Benth. ex Oerst.) Hemsl. Especie arbustiva no presente en la Península de Yucatán; se distribuye de México (Chiapas, Guerrero, Oaxaca, Queretaro, San Luis Potosí, Veracruz, Tabasco y Tamaulipas) a Panamá (excepto El Salvador).

- P. nervosa Sw. Arbusto. [Cam,Qro,Yuc].

- P. tenuifolia Sw. Arbusto. [Cam,Qro,Yuc].

* Pterocereus gaumeri (Britton \& Rose) MacDougal \& Miranda [Cactaceae]. Sinónimo de Anisocereus gaumeri (Britton \& Rose) Backeb.

$\dagger$ Punica granatum L. [Punicaceae]. [Cam,Yuc]

Randia armata (Sw.) DC. [Rubiaceae]. Determinación incorrecta de R. longiloba Hemsl. Randia armata se distribuye desde México (Sinaloa a Chiapas y Veracruz) hasta Brasil, Colombia y Las Antillas.

$R$. laetevirens Standl. Determinación incorrecta de $R$. aculeata L. Randia laetevirens se distribuye aparentemente sólo en México (Nuevo León, Querétaro, San Luis Potosí, Sinaloa, Tamaulipas y Veracruz).

Rapanea guianensis Aubl. [Myrsinaceae]. Sinónimo de Myrsine guianensis (Aubl.) Kuntze.

R. myricoides (Schltdl.) Lundell. Sinónimo de Myrsine coriacea (Sw.) R. Br. ex Roem. \& Schult.

Rauvolfia hirsuta Jacq. [Apocynaceae]. Sinónimo de R. tetraphylla L.

Rinorea guatemalensis (S. Watson) Bartlett [Violaceae]. Determinación incorrecta de $R$. hummelii Sprague. Rinorea guatemalensis se distribuye desde México (Guerrero, Oaxaca, Tabasco y Veracruz) hasta Honduras. 
Ruprechtia chiapensis Lundell ex Standl. \& Steyerm. [Polygonaceae]. Sinónimo de R. pallida Standl.

Sabal mayarum Bartlett [Arecaceae]. Sinónimo de S. yapa C. Wright ex Becc.

Salix humboldtiana Willd. [Salicaceae]. Sinónimo de S. chilensis Molina

Sapium lateriflorum Hemsl. [Euphorbiaceae]. Determinación incorrecta de S. nitidum (Monach.) Lundell, sin duda especies muy relacionadas. Sapium lateriflorum se distribuye en México (Chiapas, Guerrero, Oaxaca y Veracruz) y Belice.

S. thelocarpum Schum. \& Pittier. Determinación incorrecta de S. nitidum (Monach.) Lundell. Sapium thelocarpum presenta problemas para su delimitación específica y no es posible proporcionar su área de distribución.

* Schippia concolor Burret [Arecaceae]. No se encuentra en la porción mexicana de la Península de Yucatán; se distribuye en Belice y Guatemala.

$†$ Senna alata (L.) Roxb. [Caesalpiniaceae]. [Cam,Yuc]

S. bicapsularis (L.) Roxb. var. bicapsularis. Especie no presente en la Península de Yucatán; se distribuye exclusivamente en Sudamérica.

S. fruticosa (Mill.) Irwin \& Barneby. No se encuentra en la Península de Yucatán; se distribuye en México desde Durango y Sinaloa hasta Chiapas y en Veracruz y Tabasco.

- S. pallida (Vahl) Irwin \& Barneby var. gaumeri (Britton \& Rose) Irwin \& Barneby. Arbusto. [Cam,Qro,Yuc]

- S. pallida var. goldmaniana Irwin \& Barneby. Arbusto. [Qro].

$\dagger$ S. siamea Lam. [Qro].

$\dagger$ S. sulfurea (Collad.) Irwin \& Barneby. [Yuc]

- S. villosa (Mill.) Irwin \& Barneby. Hierba o arbusto. [Cam,Qro,Yuc]

Sickingia salvadorensis Standl. [Rubiaceae]. Sinónimo de Simira salvadorensis (Standl.) Steyerm.

Sideroxylon capiri (DC.) Pittier subsp. tempisque (Pittier) T.D. Penn. [Sapotaceae]. Especie no presente en la Península de Yucatán; se distribuye desde México (Jalisco a Chiapas y Veracruz) y Guatemala a Panamá y Las Antillas.

* S. gaumeri Pittier. Sinónimo de Sideroxylon foetidissimum Jacq. subsp. gaumeri (Pittier) T.D. Penn.

S. palmeri (Rose) T.D. Penn. Especie no presente en la Península de Yucatán; se distribuye en México por la vertiente del Pacífico desde Durango y Sinaloa hasta Chiapas y en Tamaulipas a Veracruz, Hidalgo y Puebla.

- Solanum umbellatum Mill. [Solanaceae]. Arbusto. [Qro,Yuc]

- Sophora tomentosa L. [Fabaceae]. Arbusto. [Cam,Qro,Yuc]

$\uparrow$ Spathodea campanulata P. Beauv. [Bignoniaceae]. [Cam,Qro,Yuc]

$\uparrow$ Spondias purpurea L. [Anacardiaceae]. [Cam,Qro,Yuc]

Stenocereus griseus (Haw.) Buxb. [Cactaceae]. Determinación incorrecta de S. laevigatus (Salm-Dyck) Buxb. Stenocereus griseus se distribuye desde México (naturalizada en Oaxaca, Tamaulipas y Veracruz) hasta Colombia, Venezuela y Las Antillas.

† Syzygium jambos (L.) Alston [Myrtaceae]. [Yuc]

Tabernaemontana chrysocarpa S.F. Blake [Apocynaceae]. Sinónimo de T. alba Mill.

$\dagger$ Tamarindus indicus L. [Caesalpiniaceae]. [Cam,Qro,Yuc]

$\dagger$ Tectona grandis L.f. [Verbenaceae]. [Yuc]

$\uparrow$ Terminalia catappa L. [Combretaceae]. [Cam,Qro,Yuc]

$\uparrow$ Theobroma cacao L. [Sterculiaceae]. [Yuc]

- Thevetia peruviana (Pers.) Schum. [Apocynaceae]. Arbusto. [Cam,Qro,Yuc]

Thouinia canescens Radlk. var. paucidentata (Radlk.) Votava [Sapindaceae]. En ejemplares del herbario MEXU se han encontrado determinaciones por F. V. Votava (1973) en las que se propone esta variedad; debido a que esta combinación no ha sido publicada y las dificultades para consultar la tesis doctoral de este autor, no se utiliza este nombre en el trabajo.

Thrinax parviflora Sw. [Arecaceae]. No se encuentra en la Península de Yucatán; se distribuye exclusivamente en Las Antillas.

T. wendlandiana Becc. Sinónimo de T. radiata Lodd. ex Schult. \& Schult. f.

- Tournefortia glabra L. [Boraginaceae]. Arbusto. [Cam,Qro]

* Torrubia linearibracteata (Heimerl) Standl. [Nyctaginaceae]. Sinónimo de Guapira linearibracteata (Heimerl) Lundell

* T. petenensis Lundell. Sinónimo de Guapira petenensis (Lundell) Lundell.

Trema florida Britton [Ulmaceae]. Sinónimo de T. micrantha (L.) Blume.

Trema micrantha (L.) Blume. Especie para la que se han propuesto dos variedades (T. micrantha var. micrantha y $T$. micrantha var. floridana (Britton) Standl. \& Steyerm.), las cuales aparentemente se pueden distinguir en ciertas áreas, pero que no es tan claro separar al observar toda el área de distribución de la especie. En consecuencia, en este trabajo únicamente se acepta el estatus específico.

Trichilia americana (Sessé \& Moc.) T.D. Penn. [Meliaceae]. No se encuentra en la Península de Yucatán; se distribuye desde México (desde Sonora, Durango y Sinaloa hasta Chiapas) hasta Costa Rica. 
T. arborea C. DC. Sinónimo de T. glabra L.

T. cuneata Radlk. Sinónimo de T. martiana C. DC.

T. havanensis Jacq. No se encuentra en la Península de Yucatán; se distribuye desde México (por ambas vertientes) hasta Colombia y Venezuela.

T. martiana C. DC. No se encuentra en la Península de Yucatán; se distribuye desde México (Chiapas y Veracruz) hasta Colombia, Venezuela y Brasil.

- Triumfetta semitriloba Jacq. [Tiliaceae]. Arbusto. [Cam,Qro,Yuc]

* Vernonia oolepis S.F. Blake [Asteraceae]. Sinónimo de Eremosis oolepis (S.F. Blake) Gleason.

Xylosma anisophyllum Standl. [Flacourtiaceae]. Sinónimo de X. flexuosum Kunth

X. panamense Turcz. No se encuentra en la Península de Yucatán; se distribuye desde México (Chiapas, Oaxaca, Puebla, Tabasco y Veracruz) hasta Panamá.

- Zapoteca formosa (Kunth) H.M. Hern. subsp. formosa [Mimosaceaceae]. Arbusto. [Cam,Qro,Yuc]

$\dagger$ Ziziphus jujuba (L.) Lam. [Rhamnaceae]. [Qro,Yuc]

Z. mauritana Lam. Sinónimo de Z. jujuba (L.) Lam.

\section{ESPECIES DUDOSAS}

a Acacia hindsii Benth. [Mimosaceae]. [Yuc]

a Albizia idiopoda (S.F. Blake) Britton \& Rose. [Cam,Yuc]

Alchornea latifolia Sw. [Euphorbiaceae]. [Qro]

$\checkmark$ Annona diversifolia Saff. [Annonaceae]. [Yuc]

๙ A. globiflora Schltdl. [Qro]

‘ A. purpurea Moc. \& Sessé ex Dunal. [Yuc]

*aAyenia fasciculata Millsp. [Sterculiaceae]. [Yuc]

« Bernardia oblanceolata Lundell [Euphorbiaceae]. [Qro]

$* B$. yucatanensis Lundell. [Cam]

a Bourreria huanita (DC.) Hemsl. [Boraginaceae]. Reportada para Quintana Roo; probablemente se trata de determinaciones incorrectas de B. oxyphylla Standl.

a Bursera graveolens (Kunth) Triana \& Planch. [Burseraceae]. [Qro]

Callicarpa acuminata Kunth var. pringlei (Briq.) Mold. [Verbenaceae]. Reportada para Quintana Roo; variedad con un estatus taxónomico confuso, ya que no es muy distinta de la variedad acuminata.

Cassia biflora L. [Caesalpiniaceae]. Binomio taxonómicamente ambiguo que podría asignarse a Senna angustisiliqua (Lam.) Irwin \& Barneby o Senna pallida (Vahl) Irwin \& Barneby.

a Cassipourea guianensis Aubl. [Rhizophoraceae]. [Qro]

a Cestrum racemosum Ruiz \& Pav. [Solanaceae]. [Qro]

a Cnidoscolus multilobus (Pax) I.M. Johnst. [Euphorbiaceae]. [Cam,Yuc]

a Cordia cylindrostachya (Ruíz \& Pav.) Roem. \& Schult. [Boraginaceae]. [Cam,Qro,Yuc]

$\propto$ C. elaeagnoides DC. [Qro]

* aC. serratifolia Kunth. Reportada como especie endémica para la Península de Yucatán.

a Coursetia glandulosa A. Gray [Fabaceae]. [Cam,Yuc]

a Cupania dentata DC. [Sapindaceae]. [Qro,Yuc]. Especie muy relacionada con C. glabra Sw.

a Diospyros albens Presl [Ebenaceae]. [Yuc]

a D. digyna Jacq. [Yuc]

*oD. schippii Standl. [Qro, Yuc]

a Erythrina americana Mill. [Fabaceae]. De confirmarse su presencia en el área se trataría probablemente de individuos cultivados, ya que su distribución se restringe a las partes centrales de México.

Erythroxylum areolatum L. [Erythroxylaceae]. Aparentemente presente en la Península de Yucatán, aunque los ejemplares citados para el área son determinaciones incorrectas de E. confusum Britton y E. rotundifolium Lunan.

a Eugenia itzana Lundell [Myrtaceae]. [Qro]

E. leptopa Lundell. McVaugh (1963) la indica como un probable sinónimo de E. rhombea (O. Berg) Krug \& Urb.

a E. aff. rhombea (O. Berg) Krug \& Urb. Los ejemplares citados para el área son determinaciones incorrectas de E. capuli

(Cham. \& Schltdl.) O. Berg.

*aE. tikalana Lundell. [Qro]

$* a$ E. toledinensis Lundell. [Qro]

* Guapira linearibracteata (Heimerl) Lundell [Nyctaginaceae]. Especie muy compleja taxonómicamente y que no fue posible separar de otras entidades de la familia [Qro,Yuc] 
* G. petenensis (Lundell) Lundell. Situación similar a la de G. linearibracteata.

$\propto$ Haematoxylum brasiletto $\mathrm{H}$. Karst. [Caesalpiniaceae]. [Cam,Yuc]

a Henriettea succosa (Aubl.) DC. [Melastomataceae]. [Qro]

a Hyperbaena axilliflora (Griseb.) Urb. [Menispermaceae]. Reportada de Quintana Roo; determinación dudosa ya que se trata de una especie endémica de Cuba.

$\propto H$. aff. cubensis (Griseb.) Urb. Reportada de Quintana Roo; determinación dudosa ya que se trata de una especie endémica de Cuba.

a Jatropha curcas L. [Euphorbiaceae]. [Yuc]

๘ Lonchocarpus latifolius (Willd.) Kunth [Fabaceae]. [Yuc]

a Miconia prasina (Sw.) DC. [Melastomataceae]. [Qro]

a Myrsine coriacea (Sw.) R. Br. ex Roem. \& Schult. [Myrsinaceae]. [Cam,Qro]

* Neea choriophylla Standl. [Nyctaginaceae]. Especie muy compleja taxonómicamente y que no fue posible separar de otras entidades de la familia. [Cam,Qro,Yuc]

$N$. fagifolia Heimerl. Situación similar a la de $N$. choriophylla. [Cam,Qro,Yuc]

N. psychotrioides Donn. Sm. Situación similar a la de N. choriophylla. [Cam,Qro,Yuc]

$N$. tenuis Standl. Situación similar a la de N. choriophylla. [Yuc]

a Orthion subsessile (Stand1.) Steyerm. \& Standl. [Violaceae]. [Cam]

a Piptadenia flava (Spreng. ex DC.) Benth. [Mimosaceae]. [Yuc]

a Pithecellobium tomentoum Micheli. [Yuc]

a P. tortum Mart. [Yuc]

a Pouteria sapota (Jacq.) H. Moore \& Stearn [Sapotaceae]. [Qro,Yuc]

*aRandia gaumeri Greenm. \& Thomps. [Rubiaceae]. Especie citada como endémica, pero que se encuentra en México

(Oaxaca, Tamaulipas y Yucatán), Las Antillas, Venezuela y Colombia. Es probable que se trate de un sinónimo de

$R$. obcordata S. Watson.

*aR. millspaughiana S.F. Blake.

o R. standleyana L.O. Williams. [Yuc]

\& R. xalapensis M. Martens \& Galeotti. [Yuc]

\ Rhacoma latifolia (Sw.) Urb. [Celastraceae]. [Qro]

× Sebastiania inundata Kunth [Euphorbiaceae]. [Cam]

\$ Senna reticulata (Willd.) Irwin \& Barneby [Caesalpiniaceae]. [Yuc]

a Sinclairia deamii (B.L. Rob. \& Bartlett) Rydb. [Asteraceae]. [Cam]

a Solanum atitlanum Roem. [Solanaceae]. [Qro]

a s. chiapasense K.E. Roe. [Qro]

a $S$. rudepeannum Dunal. [Cam,Qro,Yuc]

a $S$. rugosum Dunal. [Qro,Yuc]

a S. yucatanum Standl. [Yuc]

a Urera caracasana (Jacq.) Griseb. [Urticaceae]. [Cam,Yuc]

a Vitex cooperi Standl. [Verbenaceae]. [Qro]

œ Zanthoxylum mayanum Standl. [Rutaceae]. [Yuc]

$\ltimes$ Z. trichilioides Standl. [Yuc]

aygia cognata (Schltdl.) Britton \& Rose [Mimosaceae]. [Qro]

\section{LITERATURA CITADA}

Alvarez M Jr. 1961. Provincias fisiográficas de la República Mexicana. Boletín de la Sociedad de Geología de México 24: 1-20.

Antonio OR, Sousa M. 1991. Contribuciones al conocimiento del género Diphysa (Leguminosae) para la flora Mesoamericana. Anales del Instituto de Biología de la Universidad Nacional Autónoma de México, Serie Botánica 62: 115-120.

Arellano-Guillermo A, Serrano-Islas MA. 1993. Reserva de Dzilam, Yucatán. En: Salazar-VallejoSI, GonzálezNE, edrs. Biodiversidad marina y costera de México. Comisión Nacional para el Cono- cimiento y Uso de la Biodiversidad y Centro de Investigaciones de Quintana Roo. México, D.F., 630-640.

Arroyo KMT. 1976. The systematics of the legume genus Harpalyce (Leguminosae: Lotoideae). Memoirs of the New York Botanical Garden 26: 1-80.

Badillo VM. 1971. Monografía de la familia Caricaceae. Universidad Central de Venezuela. Facultad de Agronomía. Maracay.

Bailey LH. 1949. Manual of cultivated plants. MacMillan Publishing Co, New York.

Barrera A. 1962. La Península de Yucatán como provincia biótica. Revista Sociedad Mexicana de Historia Natural 23: 71-105. 
Bequaert JC. 1933. Botanical notes from Yucatan. En: Shattuck GC, edr.The Peninsula de Yucatan. Medical, biological, meteorological and sociological studies. Carnegie Institution of Washington. Washington, 505-523.

Berg CC. 1972. Olmedieae, Brosimeae (Moraceae). Flora Neotropica. Monograph 7. Organization for Flora Neotropica. New York Botanical Garden New York.

Bradburn AS, Darwin SP. 1982. An annotated checklist of plants. En: Thien LB, Bradburn AS, Welden AL, edrs. The woody vegetation of Dzibilchaltún. A Maya archeological site in northwest Yucatan, Mexico. Middle American Research Institute. The Meriden Gravure Company, 19-24.

Bravo-Hollis H. 1978. Las cactáceas de México. UNAM. Vol. 1. México, D. F.

Brummitt RK, Powell CE. 1992. Authors of plant names. Royal Botanical Gardens, Kew. Whitstable Litho Ltd., Kent.

Burger WC. 1962. Studies in New World Moraceae: Trophis, Clarisia, Acanthinophyllum. Annals of the Missouri Botanical Garden 49: 1-34.

Burger W. 1977. Moraceae. Flora Costaricensis. Fieldiana: botany 40: $94-217$.

Burkart A. 1976. A monograph of the genus Prosopis (Leguminosae subfam. Mimosoideae). Journal of the Arnold Arboretum 57: 219-249, 450-525.

Cabrera CEF. 1992. La flora de Isla Mujeres, Quintana Roo, México. Tesis Profesional. Fac. de Ciencias, UNAM. México, D. F.

Cabrera CEF, Sousa SM, Téllez VO. 1982. Imágenes de la flora quintanarroense. Centro de Investigaciones de Quintana Roo e Instituto de Biología, UNAM. México, D. F.

Cabrera CEF, Torres PSA, Salazar GVC, Sánchez SO, Serralta PL, Herrera EP. 1991. Lista florística del sureste de Quintana Roo.En: Camarena-Luhrs T, Salazar-Vallejo S, edrs. Estudios ecológicos preliminares de la zona Sur de Quintana Roo. Centro de Investigaciones de Quintana Roo. Chetumal, Quintana Roo. México, 11-19.

Campbell DG. 1988. The importance of floristic inventory in the tropics. En: Campbell, DG, Hammond, HD, edrs. Floristic inventory of tropical countries: the status of plant systematics, collections, and vegetation, plus recomendations for the future. The New York Botanical Garden, New York, 5-30.

Cannon MJ, Cannon JFM. 1989. Central American Araliaceae- a precursory study for the Flora Mesoamericana. Bulletin of the British Museum Natural History Botany 19: 5-61.

Cowan CR. 1967. Swartzia (Leguminosae, Caesalpinoideae, Swartzieae). Flora Neotropica. Monograph 1. Organization for Flora Neotropica. New York Botanical Garden, New York.

Croat TB. 1976. Sapindaceae. En: Woodson RE, Schery RW, edrs. Flora of Panama. Annals of the Missouri Botanical Garden 63: 419-540.

Cronquist A. 1981. An integrated system of classification of flowering plants. Columbia University Press, New York.

Dahlgren RMT, Clifford HT, Yao PF. 1985. The families of the Monocotyledons. Springer-Verlag. Berlin.

Daniel TH. 1988. A systematic study of Bravaisia (Acanthaceae). Proceedings of the California academy of sciences 45: 111-131.

Delgadillo MC. 1984. Mosses of the Yucatan Peninsula, Mexico. III Phytogeography. Bryologist 87: 12-16.

De Wolf GP Jr. 1960. Ficus. Annals of the Missouri Botanical Garden 47: 160 .

Durán GR. 1987. Descripción y análisis de la estructura y composición de la vegetación de los petenes del noroeste de Campeche. Biótica 12: 181-192.
Durán GR. 1995. Diversidad florística de los petenes de Campeche. Acta Botánica Mexicana 31: 73-84.

Durán GR, Olmsted I. 1987. Plantas vasculares de Sian Kaán. Listado florístico de la reserva de Sian Kaán. Amigos de Sian Kaán, Puerto Morelos, Quintana Roo, México.

Edwin G, Hou D. 1975. Celastraceae. En: Woodson RE, Schery RW, edrs. Flora of Panama. Annals of the Missouri Botanical Garden 62: 45-56.

Elias TS. 1976. A monograph of the genus Hamelia (Rubiaceae). Memoirs of the New York Botanical Garden 26: 81-144.

Espejel I. 1984. La vegetación de las dunas costeras de la Península de Yucatán. I. Análisis florístico del estado de Yucatán. Biótica 9: 183-210.

Espejel I. 1986. La vegetación de las dunas costeras de la Península de Yucatán. II. Reserva de la Biosfera Sian Ka’an, Quintana Roo, México. Biótica 11: 7-24.

Espejel I. 1987. A phytogeographical analysis of coastal vegetation in the Yucatan Peninsula. Journal of Biogeography 14: 499-519.

Espejo SA. 1991a. Hernandiaceae. Flora de Veracruz. Fascículo 67. Instituto de Ecología A. C. y University of California. Xalapa, Veracruz, México.

Espejo SA. 1991b. Notas sobre el género Gyrocarpus (Hernandiaceae) en México; un nombre nuevo Gyrocarpus mocinnoi Espejo. Acta Botánica Mexicana 13: 39-51.

Estrada-Loera, E. 1991. Phytogeographic relationships of the Yucatán Peninsula. Journal of Biogeography 18: 687-697.

Fay JJ. 1980. Nyctaginaceae. Flora de Veracruz. Fascículo 13. Instituto Nacional de Investigaciones sobre Recursos Bióticos. Xalapa, Veracruz, México.

Fernández NR. 1993. La familia Rhamnaceae en México. Tesis de Doctorado. Escuela Nacional de Ciencias Biológicas, IPN. México, D. F.

Ferrusquía-Villafranca I. 1993. Geology of Mexico: a synopsis. En: Ramamoorthy TP, Bye R, Lot A, Fa J, edrs. Biological diversity of Mexico: origins and distribution. Oxford University Press, New York, 80-86.

Flores GJS. 1983. Vegetación insular de la Península de Yucatán. Boletín de la Sociedad Botánica de México 45: 23-37.

Flores VOA. 1991. Análisis de la distribución de la herpetofauna de México. Tesis de Doctorado. Facultad de Ciencias, UNAM. México, D. F.

Fryxell PA. 1988. Malvaceae of Mexico. Systematic Botany Monographs Vol. 25.

Gallardo HC. 1992. Contribución al estudio de la flora y la vegetación del parque ecológico «La Vainilla», Zihuatanejo, Guerrero. Tesis Profesional. Facultad de Ciencias, UNAM. México, D. F.

García E. 1988. Modificaciones al sistema de clasificación climática de Köpen (para adaptarlo a las condiciones de la República Mexicana). Offset Larios, México, D. F.

García-Mendoza A. 1995. Riqueza y endemismos de la familia Agavaceae en México. En: Linares E, Dávila P, Chiang F, Bye R, Elias TS, edrs. Conservación de plantas en peligro de extinción: diferentes enfoques. UNAM. México, D. F., 51-74.

Gentry AH.1980. Bignoniaceae. Part I (Crescentieae and Tourrettieae). Flora Neotropica. Monograph 25. Organization for Flora Neotropica. New York Botanical Garden New York.

Gentry AH. 1982. Bignoniaceae. Flora de Veracruz. Fascículo 24. Instituto Nacional de Investigaciones sobre Recursos Bióticos. Xalapa, Veracruz, México.

Gentry AH. 1992. Bignoniaceae. Part II (Tribe Tecomeae). Flora Neotropica. Monograph 25(II). Organization for Flora Neotropica. New York Botanical Garden New York. 
Goldman EA, Moore RT. 1946. The biotic provinces of Mexico. Journal Mammalogy. 26: 347-361.

Grether R, Camargo RSL. 1993. Mimosa bahamensis (Leguminosae) en la Península de Yucatán, México. Boletín de la Sociedad Botánica de México 53: 55-72.

Hamilton CW. 1989a. A revision of Mesoamerican Psychotria subgenus Psychotria (Rubiaceae), Part I: Introduction and species 1-16. Annals of the Missouri Botanical Garden 76: 67-111.

Hamilton CW.1989b. A revision of Mesoamerican $P$ sychotria subgenus Psychotria (Rubiaceae), Part II: Species 17-47. Annals of the Missouri Botanical Garden 76: 386-429.

Hamilton CW.1989c. A revision of Mesoamerican Psychotria subgenus Psychotria (Rubiaceae), Part III: Species 48-61 and appendices. Annals of the Missouri Botanical Garden 76: 886-916.

Hengeveld R. 1990. Dynamic biogeography. Cambridge University Press, Cambridge.

Hekking WHA. 1988. Violaceae Part I. Rinorea and Rinoreocarpus. Flora Neotropica. Monograph 46. Organization for Flora Neotropica. New York Botanical Garden New York.

Hernández HM. 1989. Systematics of Zapoteca (Leguminosae). Annals of the Missouri Botanical Garden 76: 781-862.

Hernández SLG. 1993. Cladistic analysis of the American genera of Asparagales and the systematic study of Beaucarnea (Nolinaceae) and Hemiphylacus (Hyacinthaceae). Tesis de Doctorado. University of Texas, Austin, Texas.

Hernández-X E. 1959. La agricultura en la Península de Yucatán. En: Beltrán E, edr. Los recursos naturales del sureste y su aprovechamiento. Instituto Nacional de Recursos Naturales Renovables. Vol. III. México, D. F., 3-57.

Hodel DR. 1992. Chamaedorea palms. The species and their cultivation. The International Palm Society. Allen Press, Lawrence, Kansas.

Howard RA. 1959. Studies in the genus Coccoloba VII. A synopsis and key to the species in Mexico and Central America. Journal of the Arnold Arboretum 40: 176-220.

Howard RA. 1960. Coccoloba, Polygonaceae. En: Woodson RE, Schery RW, edrs. Flora of Panama. Annals of the Missouri Botanical Garden 47: 340-353.

Hubálek Z. 1982. Coefficients of association and similarity, based on binary (presence-absence) data: an evaluation. Biological Review (London) 57: 669-689.

Ibarra-Manríquez G, Wendt TL. 1992. El género Ficus, subgénero Pharmacosycea (Moraceae) en Veracruz, México. Boletín de la Sociedad Botánica de México 52: 3-29.

Irwin H, Barneby RC. 1982. The American Cassiinae. A synoptical revision of Leguminosae, tribe Cassieae, subtribe Cassinae in the New World. Memoirs of the New York Botanical Garden 35: 1-918.

Janson S, Vegelius J. 1981. Measures of ecological association. Oecologia 49: 371-376.

Johnston MC. 1963. The species of Ziziphus indigenous to United States and Mexico. American Journal of Botany 50: 10201027.

Johnston MC. 1971. Revision of Colubrina (Rhamnaceae). Brittonia 23: $2-53$.

Kaastra RC.1982. Pilocarpinae(Rutaceae).Flora Neotropica. Monograph 33. Organization for Flora Neotropica. New York Botanical Garden, New York.

King RM, Robinson H. 1987. The genera of the Eupatorieae (Asteraceae). Systematic Botany Monographs Vol. 22.

Krukoff BA. 1982. Notes of the species of Erythrina. XVIII.Allertonia 3: $121-138$.
Landrum LR.1992. Mosiera (Myrtaceae) in Mexico and Mesoamerica. Novon 2: 26-29.

Lay KK. 1950. The American species of Triumfetta L. Annals of the Missouri Botanical Garden 37: 315-395.

León J. 1987. Botánica de los cultivos tropicales. Instituto Interamericano de Cooperación para la Agricultura. San José, Costa Rica.

Leopold AS. 1990. Fauna silvestre de México. Instituto Mexicano de Recursos Naturales Renovables. México, D. F.

Liogier HA, Martorell LF. 1982. Flora of Puerto Rico and adjacent islands: a systematic synopsis. Universidad de Puerto Rico. Puerto Rico, Puerto Rico.

Lott EJ. 1987. Nota sobre la distribución de Savia sessiliflora (Euphorbiaceae) en México. Boletín de la Sociedad Botánica de México 47: 90-91.

Lott EJ. 1993. Annotated checklist of the vascular flora of the Chamela Bay Region, Jalisco, México. Ocassional Papers of the California Academic Sciences No. 148.

Lundell CL. 1934. Preliminary sketch of the phytogeography of the Yucatan Peninsula. Contributions to American Archaelology 12: 257-321.

Lundell CL. 1945. The genus Cnidoscolus in Mexico: new species and critical notes. Bulletin of the Torrey Botanical Club 72: 271-294.

Lundell CL. 1960. Plantae mayanae I. Notes on collections from the lowlands of Guatemala. Wrightia 2: 51.

Lundell CL. 1962. Plantae Mayanae V. Petenea cordata, a new genus and species in the Elaeocarpaceae, and other taxonomic notes. Wrightia 3: 22.

Lundell CL, Lundell AA. 1983. The flora of northern Yucatan and the Coba area of Quintana Roo, Mexico: collections and observations in 1938. Wrightia 7: 97-228.

Martínez-García J. 1985. Achatocarpaceae. Flora de Veracruz. Fascículo 45. Instituto Nacional de Investigaciones sobre Recursos Bióticos. Xalapa, Veracruz, México.

Mathias MT, Theobald WL. 1981. A revision of the genus Hyperbaena (Menispermaceae). Brittonia 33: 81-104.

McVaugh R. 1945. The genus Jatropha in America: principal intrageneric groups. Bulletin of the Torrey Botanical Club 72: 271-294.

McVaugh R. 1963. Myrtaceae. En: Standley PC, Steyermark, JA, Williams LO, edrs. Flora of Guatemala. Fieldiana: Botany 24 (Part VII): 283-405.

Millspaugh CH.1895. Contribution to the flora of Yucatan. Publications of the Field Columbian Museum. Botanical series 1: 3-56.

Millspaugh CH. 1896. Contribution II to the coastal and plain flora of Yucatan. Publications of the Field Columbian Museum. Botanical series 1: 281-339.

Millspaugh CH. 1898. Contribution III to the coastal and plain flora of Yucatan. Publications of the Field Columbian Museum. Botanical series 1: 345-410.

Millspaugh CH, Chase A. 1904. Compositae. Plantae Yucatanae (regionis Antillanae). Plants of the insular, coastal and plain regions of the Peninsula of Yucatan, Mexico. Publications of the Field Columbian Museum. Botanical series 3: 85-151.

Miranda F. 1964. (Reimpresión). Estudios acerca de la vegetación. Sobretiro 2. Colegio de Postgraduados. Escuela Nacional de Agricultura. México, D.F.

Moreno-Casasola P. 1988. Patterns of plant species distribution on coastal dunes along the Gulf of Mexico. Journal of Biogeography 15: 787-806.

Moreno-Casasola P, Espejel I. 1986. Sand dune vegetation along the Gulf of Mexico and Caribean sea. Vegetatio 66: 147-182.

Moreno NP. 1980. Caricaceae. Flora de Veracruz. Fascículo 10. Instituto Nacional de Investigaciones sobre Recursos Bióticos. Xalapa, Veracruz, México. 
Nash DL, Moreno NP. 1981. Boraginaceae. Flora de Veracruz. Fascículo 18. Instituto Nacional de Investigaciones sobre Recursos Bióticos. Xalapa, Veracruz, México.

Nash DL, Nee M. 1984. Verbenaceae. Flora de Veracruz. Fascículo 41. Instituto Nacional de Investigaciones sobre Recursos Bióticos. Xalapa, Veracruz, México.

Nee M. 1984. Ulmaceae. Flora de Veracruz. Fascículo 40. Instituto Nacional de Investigaciones sobre Recursos Bióticos. Xalapa, Veracruz, México.

Nee M. 1986. Solanaceae I. Flora de Veracruz. Fascículo 49. Instituto Nacional de Investigaciones sobre Recursos Bióticos. Xalapa, Veracruz, México.

Nee M. 1993. Solanaceae II. Flora de Veracruz. Fascículo 72. Instituto de Ecología A. C. y University of California. Xalapa, Veracruz, México.

Nevling LI Jr., Barringer K. 1988. Thymelaeaceae. Flora de Veracruz. Fascículo 59. Instituto Nacional de Investigaciones sobre Recursos Bióticos. Xalapa, Veracruz, México.

Nowicke JW. 1970. Apocynaceae. En: Woodson RE, Schery RW, edrs. Flora of Panama. Annals of the Missouri Botanical Garden 57: 59-130.

Olmsted IC, Durán GR. 1986. Aspectos ecológicos de la selva baja inundable de la reserva de Sian Ka'an, Quintana Roo, México. Biótica 11: 151-179.

Orellana R, Villers L, Franco V, Ojeda L. 1985. Algunos aspectos ecológicos de los agaves de la Península de Yucatán. En: Cruz C, del Castillo L, Robert M, Ondarza RN, edrs. Biología y aprovechamiento integral del henequén y otros agaves. Centro de Investigaciones Científicas de Yucatán, A. C. Chiapas, México, 39-53.

Ortiz DJJ. 1994. Polygonaceae. Etnoflora Yucatanense. Fascículo 10. Universidad Autónoma de Yucatán. Mérida, Yucatán, México.

Pacheco L. 1981. Ebenaceae. Flora de Veracruz. Fascículo 16. Instituto Nacional de Investigaciones sobre Recursos Bióticos. Xalapa, Veracruz, México.

Pennington TD. 1981. Meliaceae. Flora Neotropica. Monograph 28. Organization for Flora Neotropica. New York Botanical Garden, New York.

Pennington TD. 1990. Sapotaceae. Flora Neotropica. Monograph 52. Organization for Flora Neotropica. New York Botanical Garden, New York.

Perry JP Jr. 1991. The pines of Mexico and Central America. Timber Press, Portland, Oregon.

Plowman T.1991.Erythroxylaceae. En: BurgerW, edr. Flora costaricencis. Fieldiana: Botany 28: 30-36.

Poppendieck HH. 1981. Cochlospermaceae. Flora Neotropica. Monograph 27. Organization for Flora Neotropica. New York Botanical Garden, New York.

Prance GT. 1972. Chrysobalanaceae. Flora Neotropica. Monograph 9. Organization for Flora Neotropica. New York Botanical Garden, New York.

Prance GT. 1989. Chrysobalanaceae. Flora Neotropica. Monograph 9S. Organization for Flora Neotropica. New York Botanical Garden, New York.

Quero HJ. 1992. Las palmas silvestres de la Península de Yucatán. Publicaciones Especiales 10. Instituto de Biología, UNAM. México, D. F.

Quero HJ, Read RW. 1986. A revision of the palm genus Gaussia. Systematic Botany 11: 145-154.

Read RW.1975. The genus Thrinax (Palmae: Coryphoideae). Smithsonian Contributions of Botany 19: 69-77.

Rico AML. 1989. Systematic study of the generic patterns in the tribe Ingeae (Leguminosae: Mimosoideae), withemphasis on ZygiaCaulanthon. Tesis (Doctorado). University of Southampton. Reino Unido.
Rico AML. 1991. New species, combinations and synonyms for Zygia, Cojoba, Marmaroxylon and Pithecellobium (Leguminosae, Mimosoideae, Ingeae). Kew Bulletin 46: 493-521.

Rico AML. 1992. Notes on Albizia niopoides (Spruce ex Benth.) Burkart (Leguminosae: Mimosoideae). Kew Bulletin 47: 699-702.

Rico AML. 1994. Nueva especie mirmecófila de Acacia (Leguminosae) de la Península de Yucatán, México. Acta Botánica Mexicana 26: 7-10.

Rico-Gray V. 1982. Estudio de la vegetación de la zona costera inundable del noroeste del estado de Campeche, México: los petenes. Biótica 7: 171-190.

Robyns, A. 1963. Essai de monographie du genre Bombax $s . l$. (Bombacaceae). Bulletin du jardin botanique de l'état. 33: 1311.

Rohwer JG. 1993. Lauraceae: Nectandra. Flora Neotropica. Monograph 60. Organization for Flora Neotropica. New York Botanical Garden, New York.

Rudd VE. 1977. The genus Machaerium (Leguminosae) in Mexico. Boletín de la Sociedad Botánica de México 37: 119-146.

Rzedowski J. 1978. Vegetación de México. Ed. Limusa. México, D. F.

Sánchez O, López G. 1988. A theoretical analysis of some indices of similarity as applied to biogeography. Folia Entomológica Mexicana 75: 119-145.

Sánchez SO, Cabrera CEF, Torres PSA, Herrera EP, Serralta PL, Salazar GVC. 1991. Vegetación. En: Camarena-Luhrs T, Salazar-Vallejo S, edrs. Estudios ecológicos preliminares de la zona Sur de Quintana Roo. Centro de Investigaciones de Quintana Roo. Chetumal, Quintana Roo. México, 31-48.

Sánchez-Vindas PE. 1990. Myrtaceae. Flora de Veracruz. Fascículo 62. Instituto de Ecología A. C. y University of California. Xalapa, Veracruz, México.

Sleumer HO. 1980. Flacourtiaceae. Flora Neotropica. Monograph 22. Organization for Flora Neotropica. New York Botanical Garden, New York.

Sleumer HO. 1984. Olacaceae. Flora Neotropica. Monograph 38. Organization for Flora Neotropica. New York Botanical Garden, New York.

Smith HM. 1941. An analysis of the biotic provinces of Mexico, as indicated by the distribution of the lizards of the genus Sceloporus. Anales de la Escuela Nacional de Ciencias Biológicas 2: 95-110.

Sosa V. 1979. Araliaceae. Flora de Veracruz. Fascículo 8. Instituto Nacional de Investigaciones sobre Recursos Bióticos. Xalapa, Veracruz, México.

Sosa V, Flores JS, Rico-Gray V, Lira R, Ortiz JJ. 1985. Lista florística y sinonimia maya. Etnoflora Yucatanense. Fascículo 10. Xalapa, Veracruz, México.

Sousa SM, Cabrera CEF. 1983. Listados florísticos de México II. Flora de Quintana Roo. Instituto de Biología, UNAM. México, D. F.

Sousa SM. 1993. El género Inga (Leguminosae, Mimosoideae) del sur de México y Centroamérica, estudio previo para la Flora Mesoamericana. Annals of the Missouri Botanical Garden 80: 233-269.

Ståhl B. 1989. A synopsis of Central American Theophrastaceae. Nordic Journal of Botany 9: 15-30.

Standley PC. 1935. New plants from the Yucatan Peninsula. Publications of the Carnegie Institution of Washington 461: 51-91.

Standley PC. 1936. Las relaciones geográficas de la flora mexicana. Anales del Instituto de Biología de la Universidad Nacional Autónoma de México 7: 9-16.

Standley PC. 1977. La Flora. Enciclopedia Yucatanense. 2da. ed. Gobierno del Estado de Yucatán. Tomo I. México, D. F.

Standley PC, Steyermark, JA, Williams LO. 1946-76. Flora of Guatemala. Fieldiana: Botany 24(1-12). 
Stearn WT. 1992. The genus Jacquinia (Theophrastaceae) in Jamaica. Nordic Journal of Botany 12: 231-238.

Steyermark JA. 1972. Rubiaceae. En: The botany of the Guayana Highland. Part IX. Memoirs of the New York Botanical Garden 23: 227-832.

Tebbs MC. 1990. Revision of Piper (Piperaceae) in the New World 2. The taxonomy of Piper section Churumayu. Bulletin of the British Museum Natural History, Botany 20: 193-236.

Tebbs MC. 1993. Revision of Piper (Piperaceae) in the New World 3. The taxonomy of Piper sections Lepianthes and Radula. Bulletin of the British Museum Natural History, Botany 23: 1-50.

Téllez VO, Cabrera CEF. 1987. Listados florísticos de México VI. Flórula de la Isla de Cozumel, Quintana Roo. Instituto de Biología, UNAM. México, D. F.

Thomas WW. 1988. A conspectus of Mexican and Central american Picramnia (Simaroubaceae). Brittonia 40: 89-105.

Todzia C. 1989. A revision of Ampelocera (Ulmaceae). Annals of the Missouri Botanical Garden 76: 1087-1102.

Trejo-Torres JC, Durán GR \& Olmsted I. 1993. Manglares de la Península de Yucatán. En: Salazar-Vallejo SI, González NE, edrs. Biodiversidad marina y costera de México. Comisión Nacional para el Conocimiento y Uso de la Biodiversidad y Centro de Investigaciones de Quintana Roo. México. D. F., 660-672.

Villanueva GR, Cabrera CEF. 1990. Nuevos registros florísticos para la reserva de la biosfera de Sian Ka'an, Quintana Roo, México. En: Navarro D, Robinson JG, edrs. Diversidad biológica en la reserva de la biosfera de Sian Ka'an, Quintana Roo, México.
Centro de Investigaciones de Quintana Roo y University of Florida. Chetumal, Quintana Roo, México, 95-98.

Villaseñor JL. 1989. Manual para la identificación de las Compositae de la Península de Yucatán y Tabasco. Rancho Santa Ana Botanic Garden Technical Report No. 4. Claremont, California.

Webster GL. 1992. Revision of Astrocasia (Euphorbiaceae). Systematic Botany 17: 311-323.

Webster GL, Huft MJ. 1988. Revised synopsis of panamian Euphorbiaceae. Annals of the Missouri Botanical Garden 75: 1087-1144.

Woodson RE Jr. 1938. Studies in the Apocynaceae VII. An evaluation of the genera Plumeria L. and Himatanthus Willd. Annals of the Missouri Botanical Garden 25: 189-224.

Woodson RE Jr., Schery RW. 1950. Leguminosae subfamily Mimosoideae. Annals of the Missouri Botanical Garden 37: 184-314.

Woodson RE Jr., Schery RW. 1961. Nyctaginaceae. Annals of the Missouri Botanical Garden 48: 51-66.

Zárate PS. 1994. Revisión del género Leucaena en México. Anales del Instituto de Biología de la Universidad Nacional Autónoma de México, Serie Botánica 65: 83-162.

Zona S. 1990. A monograph of Sabal (Arecaceae: Coryphoideae). Aliso 12: 583-666.

Zarucchi JL. 1993. Catalogue of the Flowering Plants and Gymnosperms of Peru. En: Brako L, Zarucchi JL, edrs. Monographs in Systematic Botany from the Missouri Botanical Garden. Vol. 45: 719-728. 